\title{
Research Paper \\ The effectiveness of unified transdiagnostic therapy on strategies cognitive emotion regulation in mothers of children with cancer
}

\author{
MohammadSaleh Ashena ${ }^{1}$, MohammadAli Besharat ${ }^{2}$, Saied Malihialzuckerini ${ }^{3}$, Zohreh Rafezi ${ }^{4}$ \\ 1. Ph.D in Health Psychology, Department of Health Psychology, Karaj Branch, Islamic Azad University, Karaj, Iran. \\ 2. Professor,Department of Psychology, University of Tehran, Tehran, Iran. \\ 3. Assistant Professor, Department of Health Psychology, Karaj Branch,Islamic Azad University, Karaj, Iran. \\ 4. Assistant Professor, Department of Clinical Psychology, Allameh Tabataba'i University, Tehran, Iran.
}

Citation: Ashena M.S, Besharat M.A, Malihialzuckerini S, Rafezi Z. The effectiveness of unified transdiagnostic therapy on strategies cognitive emotion regulation in mothers of children with cancer. $\mathrm{J}$ of Psychological Science. 2021; 20(104): 1383-1402.

URL: https://psychologicalscience.ir/article-1-1104-fa.html
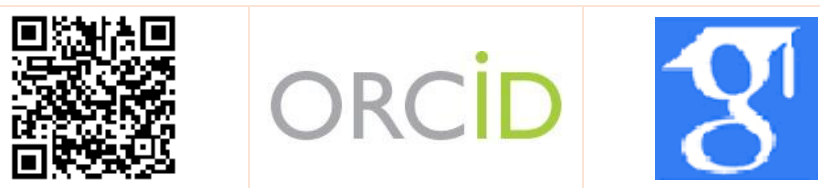

$\underline{10.52547 / J P S .20 .104 .1383}$
A R T I C L E I N F O

Keywords:

Unified transdiagnostic therapy,

Cognitive

Regulation, mother of children with cancer

Received: 05 Jan 2021

Accepted: 24 Jan 2021

Available: 23 Oct 2021
A B S T R A C T

Background: Studies have shown that Unified transdiagnostic therapy is unique in this emphasis on how to experience and respond to emotions. However, there is not any research on the effectiveness of Unified transdiagnostic therapy on the Cognitive Emotion Regulation in mothers of children with cancer so far.

Aims: The purpose of this study was to determine the effectiveness of Unified transdiagnostic therapy on Cognitive Emotion Regulation in mothers of children with cancer.

Methods: This research was a quasi-experimental study with pre-test and post-test with the control group. The statistical population consisted of all mothers of children with cancer who were referred to Mahak, Shohada Tajrish, Bahrami, Hazrate Ali Asghar hospitals, in 2019 in Tehran. 31 mothers were selected by available sampling method and randomly assigned to 15 cases in the experimental group and 16 cases in the control group. The present research tools are the Cognitive Emotion Regulation Questionnaire (CERQ) short form (Garnefski \& Kraaij,2006) and Unified Transdiagnostic Therapy (Barlow, 2011). The experimental group received the Unified Transdiagnostic Therapy for 12 sessions, each for 90 minutes. Data analysis was performed by the univariate analysis of covariance analysis method and using SPSS23 software.

Results: The results showed Unified transdiagnostic therapy in the experimental group has increased using adaptive strategies compared to the control group include acceptance, positive refocusing, refocus on planning, positive reappraisal, and putting into perspective and also, it has led to a significant reduction in using maladaptive strategies include, self-blame, rumination, catastrophizing, and other-blame $(\mathrm{P} \leq 0.001)$.

Conclusion: Unified transdiagnostic therapy increases using adaptive strategies and decreases using maladaptive strategies in mothers of children with cancer that this process will help improve the mental health of these mothers.

* Corresponding Author: MohammadAli Besharat, Professor,Department of Psychology, University of Tehran, Tehran, Iran.

E-mail: besharat@ut.ac.ir

Tel: (+98) 2161117488

2476-5740/ ( 2021 The Authors. This is an open access article under the CC BY-NC-ND license

(https://creativecommons.org/licenses/by-nc/4.0/). 


\section{Extended Abstract}

\section{Introduction}

The World Health Organization estimates that by 2022, approximately 15 million people worldwide will be affected by cancer, and more than $80 \%$ of them will become long-term survivors of their disease. They will experience pain and other negative experiences during their illness (Duffy et al., 2019). Advanced treatment for pediatric cancer is often associated with two to three years of frequent and prolonged hospitalization. This process creates an extremely stressful experience for the whole family. Having a child with cancer may lead to a family crisis or threaten its integrity. Some families are well adapted to this situation, while others experience severe problems. For example, divorce and disruption of daily family affairs (Zhang et al., 2019). Parents of children with cancer face emotional, psychological and mental challenges in order to cope with their child's illness (Majdalani et al., 2018).The greater the negative impact of the child's condition on his family life, the greater the impact on the psychological performance of parents, especially mothers. Research has shown that high levels of care burden, remain for years after treatment is completed. Symptoms of psychological distress, such as symptoms of posttraumatic stress, loneliness, helplessness, and increased insecurity, have also been reported in parents of children with cancer (Kucukoglu, Polat, Tufekci \& Bulu, 2016). The burden of care is defined as the sensory, psychological and social response of the caregiver (Zarit, 2004). In addition, timely identification of care burden, in caregivers has a crucial role in promoting their health, because burden of care is the most important factor determining the quality of life of caregivers. On the other hand, there is a direct and significant relationship between the quality of life of children and mothers (Klassen et al., 2011). Mothers of children with cancer are often afraid to experience their own severe negative emotional states, which is understandable because their negative emotional states are often very strong, although their efforts to avoid these states often have inconsistent consequences And increases negative emotional states in them (Baer, 2003).
Emotion regulation is the ability to adjust the parameters of an emotional experience, which includes two unique non-reciprocal responses. Selfregulation of emotions is a rapid and reactive reaction to the onset of an emotional challenge, while conscious emotion regulation is a long-term, more obvious reciprocal reaction. The ability to perform conscious emotion regulation, mind holding, and conscious control is an important skill (Grabell et al., 2019).Emotion regulation involves the use of behavioral and cognitive strategies to change the duration or intensity of an emotion experience (Kring and Sloan, 2009). Cognitions or cognitive processes help people regulate their emotions and feelings and not be overwhelmed by the intensity of emotions (Gross and Thompson, 2007).

In this regard, Garnefski, Kraaij and spinhoven (2001) proposed nine different strategies for cognitive emotion regulation, which are divided into two categories: adaptive (positive) and maladaptive (negative) strategies. Adaptive strategies include: acceptance (thinking with acceptance content), positive reappraisal(focusing on the positive side of events), refocusing on planning (thinking about the stages of overcoming or changing the negative event), positive refocusing(thinking about the aspect Positive aspects of the event or personal promotion), putting into perspective (thinking about the insignificance of the event or emphasizing its relativity compared to other events). Inconsistent strategies also include: self-blame (thinking with the content of blaming and self-blame), other-blame (thinking with the content of blaming and blaming others for what happened), mental rumination (mental preoccupation with feelings and thoughts related to the event Negative) and catastrophizing(thinking with the content of horror of the accident). Research has shown that using different strategies has different consequences. It seems that overuse of incompatible strategies or underuse of adaptive strategies can be the underlying causes of emotional problems. Incompatible emotion regulation strategies increase the risk of emotional problems and psychological damage, while adaptive strategies act as a protective factor (Yaghoubi and Waqef, 2019, Ashena et al.,2016). 


\section{Monthly Journal of Psychological Science}

One of the useful therapies in the field of emotion regulation is Integrated transdiagnostic treatment, which has been introduced in response to the limitations of specific cognitive-behavioral therapies, in which the same principles and treatment protocols are used for different types of emotional disorders. An integrated treatment protocol is designed for people with emotional disorders, especially those with mood and anxiety disorders, and is rooted in a cognitivebehavioral tradition, and is a transdiagnostic cognitive-behavioral therapy focused on emotion. Although it has a cognitive-behavioral tradition, in its special emphasis on how to experience and respond to their emotions in people with emotional disorders, it is unique. This treatment emphasizes the adaptive and functional nature of emotions and mainly tries to identify and correct non-adaptive attempts to regulate emotional experiences, thereby facilitating appropriate processing. Also disproportionate emotional response to internal symptoms (visceral) And extinguishes external signs (Farchione et al., 2012). The rationale for the formation of transdiagnostic therapies is based on theoretical concepts and experimental results regarding the existence of common factors between emotional disorders, which were mainly designed to target these causative factors (Boisseau, Farchione, Fairholme, Ellard \& Barlow, 2010).Transdiagnostic protocols are designed to target cognitive and behavioral processes involved in a wide range of psychological disorders, including Integrated transdiagnostic Treatment Protocol(Barlow, 2011), which is designed for people with emotional disorders. The high rate of association between these disorders and the high rate of recovery in disorders associated with the main disorder being treated is the reason for transdiagnostic treatment. The Integrated Transdiagnostic Therapy Protocol is a coping-based cognitive-behavioral therapy that focuses primarily on changing nonconforming responses to emotional experiences. This treatment tries to target the main processes that cause emotional disorders by integrating the common components of cognitive-behavioral therapy for emotional disorders and using the latest advances in the field of emotion science and third wave therapies.
Vol. 20, No. 104, Autumn(November) 2021

(Boisseau, Farchione, Fairholme, Ellard \& Barlow, 2010).

With this explanation, the lack of research on the effect of this treatment on mothers with children with cancer can be felt more than ever. Parents of children with cancer, especially mothers, due to the burden of care and more emotional interaction with children, as well as the stress of the imposed conditions of the child's illness, suffer from cognitive dysfunction and as a result of non-functional and disruptive exposure to the acute issue of the child., Causes more problems for both parent and child (Domit, Rahi, Saab and Majdalani, 2018).Due to the special emphasis of this treatment on emotions, in this study, an attempt has been made to evaluate its different dimensions by examining the effectiveness of integrated transdiagnostic treatment on "cognitive emotion regultion" strategies in the community of mothers with children with cancer. Given the above, the present study seeks to answer the question of whether integrated transdiagnostic therapy has an effect on cognitive emotion regulation strategies of mothers of children with cancer?

\section{Method}

The present study was a quasi-experimental study with a pretest-posttest design with a control group. The statistical population of the present study was the mothers of children with cancer in Tehran who referred to hospitals in the city in 1398, which according to the integrated statistical system of Mahak Hospital was 10460 patients. At first, 36 people were selected by available sampling method in accordance with the inclusion criteria and were randomly assigned to the experimental and control groups. After the sample fell, 31 people (15 in the experimental group, 16 in the control group) Remained in research. The sample group included 12 people from Mahak Hospital, 6 people from Shohada Tajrish Hospital, 7 people from Bahrami Hospital and 6 people from Hazrat Ali Asghar Hospital. The criteria for entering the study were: 1- Having informed consent. 2- The age of the children, which should have been in the age range of 7 to 12 years. 3 . Children should have received specialized medical treatment for cancer at the time of the study. Exclusion criteria were: 1- Completion or suspension 


\section{Monthly Journal of Psychological Science}

of the treatment process of children and their discharge from the hospital. 2- Other family members do not have cancer or other chronic diseases. 3Absence from more than two sessions or Withdrawal of sample members from the treatment process. Also, in order to ensure the compliance of the inclusion criteria of the sample members, in addition to the researcher examining the psychological and family conditions, the medical records of the children of these mothers were reviewed by a specialist physician. In order to observe the ethical principles of confidentiality in the research, all participants were assured that the information will be collected for research purposes and the information of the respondents will be confidential. They were also reminded that they could be informed of the research results if they wished. Also, according to the ethical principles of justice in the research, the control group was assured that after the end of the research process, they will be offered the desired psychological treatment if they wish. The research method was that first both groups answered the questionnaire of cognitive emotion regulation strategies before any intervention in the same conditions. Then, the intervention based on integrated meta-diagnostic treatment was performed as a twelve-session program including two sessions per week for the experimental group. The duration of each session was 90 minutes. The control group did not receive any intervention. At the end of the training, both groups answered the post-test questionnaires. In the present study, integrated meta-diagnostic intervention was applied as an independent variable and cognitive emotion regulation was applied as a dependent variable. Data analysis was performed by univariate analysis of covariance using SPSS software version 23.

\section{Results}

The results show that after the integrated transdiagnostic intervention in mothers of children with cancer, in the experimental group, the mean of components of adaptive strategies increased significantly. Also the maladaptive components have decreased on average. These changes are not observed in the control group. For a more detailed study, we will make a statistical inference of the
Vol. 20, No. 104, Autumn(November) 2021

significant difference between the pre-test and posttest of the experimental and control groups.

To test this hypothesis and control the differences of the subjects in the pre-test, one-way analysis of covariance was used. First, to test the assumptions of analysis of covariance, the results of KolmogorovSmirnov test to check the normality of the data, Levene test to measure the equality of variance and homogeneity of regression slope were performed, and then a table of effect tests between subjects was presented. The Kolmogorov-Smirnov test was used to investigate the assumption that the distribution of scores of variables was normal. Due to the fact that the significance level in the mentioned test was greater than 0.05 , it can be concluded that the distribution of scores of dependent variables is normal $(p \leq 0.05)$. Also, according to the results of Levene test and since the significance level of $F$ statistic of all components was greater than 0.01 , so the error variance of the two groups is equal and no difference is observed between them. Also, the value of $F$ is not significant for the variables $(p \leq 0.05)$. Therefore, it can be concluded that the null hypothesis is not rejected and the regression homogeneity slope assumption is observed. To find out which variable or variables differ between the two groups, one-way analysis of covariance for the effectiveness of integrated transdiagnostic treatment was performed separately, the results of which are shown in Table 3 . The results show that the $\mathrm{F}$ value calculated for the subscales of cognitive emotion regulation, self-blame $(\mathrm{P} \leq 0.001, \mathrm{~F}=48.13)$, acceptance $(\mathrm{P} \leq 0.001, \mathrm{~F}=$ 45.32), rumination $(\mathrm{P} \leq 0.001, \mathrm{~F}=25 / 14)$, Positive refocusing $(\mathrm{P} \leq 0.001, \mathrm{~F}=31.33)$, Planning refocusing $(\mathrm{P} \leq 0.001, \mathrm{~F}=66.36)$, positive reappraisal $(\mathrm{P} \leq 0.001$, $\mathrm{F}=43 / 86)$, putting into perspective $(\mathrm{P} \leq 0.001$, $\mathrm{F}$ $=34 / 66)$, catastrophizing $(\mathrm{P} \leq 0.001, \mathrm{~F}=54.88)$ and blaming others ( $\mathrm{P} \leq 0.001, \mathrm{~F}=49 / 27)$. Considering that it is $(\mathrm{P} \leq 0.5)$, the calculated value of $\mathrm{F}$ is significant, which indicates the difference between the effect of the test conditions on the dependent variable (positive and negative components of cognitive emotion regulation).

\section{Conclusion}

The main purpose of this study was the effectiveness of integrated transdiagnostic therapy on cognitive 
emotion regulation of mothers with children with cancer. According to the research findings, the mean post-test scores of the experimental group in the subscales of the Cognitive Emotion Regulation Questionnaire were significantly different from the control group. In other words, integrated transdiagnostic intervention has had a significant effect on increasing the use of adaptive strategies and reducing the use of maladaptive strategies. These findings are consistent with the results of the research of Kamacho et al. (2019), Sakiris \& Berle(2019), Sherman and Ernrich (2019), Jarvy Steele et al., (2018) and Varkovitzky \& Sherrill, (2018). The results of this study are in line with the results of the studies of the mentioned researches, including: the effect of integrated transdiagnostic therapy on reducing anxiety symptoms, increasing the use of adaptive emotional regulation strategies and reducing the use of maladaptive emotional regulation strategies, reducing symptoms of emotional disorders, depressive symptoms and emotional regulation problems. The trasdiagnostic approach targets the underlying components of psychological trauma and can be applied to disorders and problems that have the same underlying underpinning. This treatment for people with emotional and cognitive disorders, especially people with anxiety and mood disorders can be an economical and powerful option compared to other diagnostic treatment protocols (Ashena, Besharat, Malihi Al-zuckerini and Rafezi,
2020). As can be seen, this therapeutic approach has a significant effect on the improvement of emotional disorders and emotion regulation (Farchione, Fairholme, Ellard, Boisseau, Thompson-Hollands \& Carl, 2012). The transdiagnostic approach has received special attention despite the short time that has elapsed since its research and clinical application (Harvey, Watkins, Mansell \& Shafran, 2004). Transdiagnostic therapies have been shown to be effective in treating emotional disorders (Norton, Hayes \& Springer, 2008).

\section{Ethical Considerations}

Compliance with ethical guidelines: This article is taken from the doctoral dissertation of the first author, from the Islamic Azad University, Karaj Branch in the field of health psychology. The approval date of this plan is 31/12/2018 and its research code is 950336194. The code of ethics of this research (IR.IAU.K.REC.1398.071) on 4/9/2019 from the National Ethics Committee in Biomedical Research of the Ministry Health, treatment and medical education of Iran, was obtained.

Funding: This research is in the form of a doctoral dissertation without financial support.

Authors' contribution: The first author is the main researcher of this research. The second author is the supervisor and the third and fourth authors are the doctoral dissertation advisor professors.

Conflict of interest: The authors have no conflict of interest in this study.

Acknowledgments: This study was a small empathy and support for children with cancer and their families. I sincerely thank them and all those who helped in this research, and I also wish them good health. 


\section{اثربخشى كروه درمانى فراتشخيصى يكيارجه بر راهبردهاى تنظيم شناختى هيجان مادران كود كان مبتلا به سرطان}

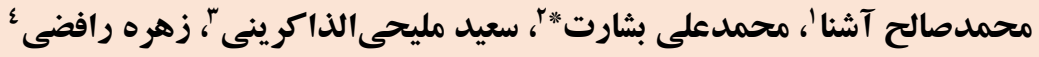

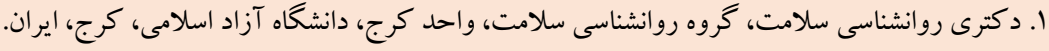

r. استاد، كروه روانشناسى، دانشگاه تهران، تهران، ايران.

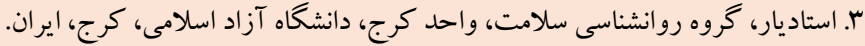

F. استاديار، كروه روانشناسى بالينى، دانشكاه علامه طباطبايى، تهران، ايران.

جكيده

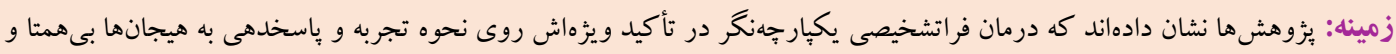

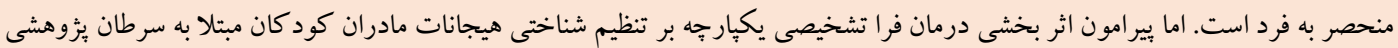

$$
\text { صورت نكرفته است. }
$$

هدف: اين يُزوهش با هدف تعيين اثربخشى درمان فرا تشخيصى يكهارجه بر تنظيم شناختى هيجان مادران كود كان مبتلا به سرطان انجام شد.

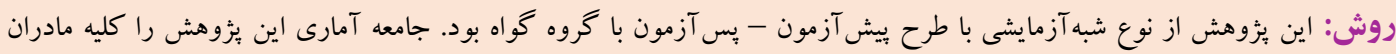
كود كان مبتلا به سرطان كه در بيمار ستانهاى محك،، شهداى تجريش، بهر امى و حضرت على اصغر در سال ^وس ا در شهر تهران تحت درمان

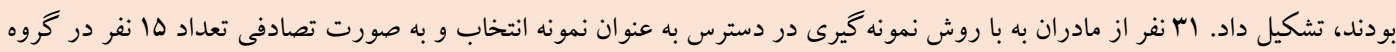

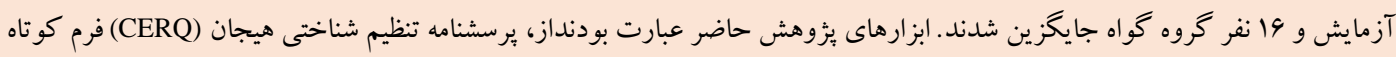

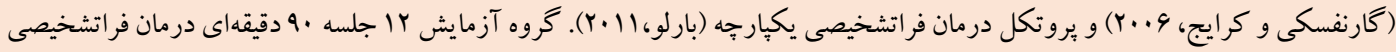

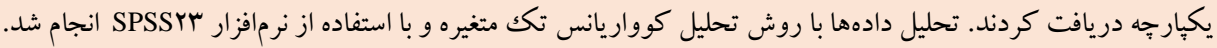

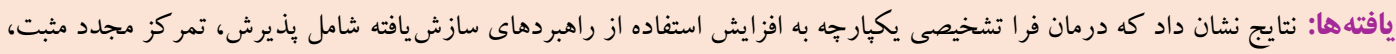

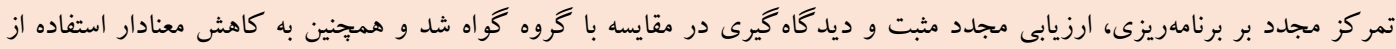

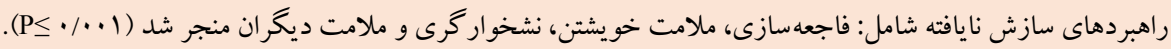
نتيجه كيرى: درمان فرا تشخيصى يكِياجهنگ باعث افزايش استفاده از راهبردهاى انطباقى هيجانى و كاهش استفاده از راهبردهاى غير

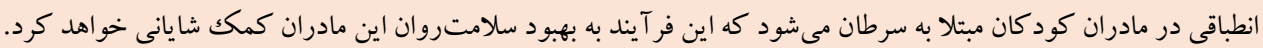

مشخصات مقاله

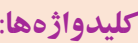

درمان فراتشخيصى يكِارجه، تنظيم شناختى هيجان، مادران كود كان مبتلا به سرطان هيجان

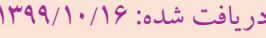
يذّيرفته شده: ه•/||/

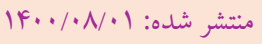

* نويسنده مسئول: محمدعلى بشارت، استاد، كروه روانشناسى، دانشگاه تهران، تهران، ايران.

رايانامه: besharat@ut.ac.ir

تلفن: 
كود كان مبتلا به سرطان گزارش مىشود (كيجكولدو، بولات، توفكجى و

dolقo

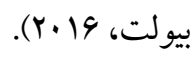

البته در سى سال اخير ييشرفت در زمينه درمان سرطان كودكان موجب

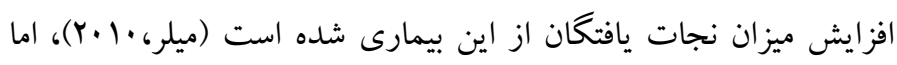

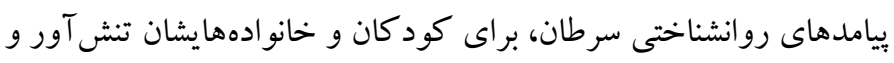

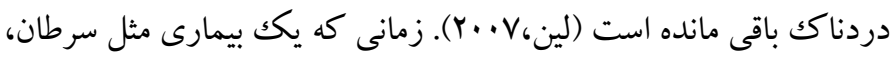

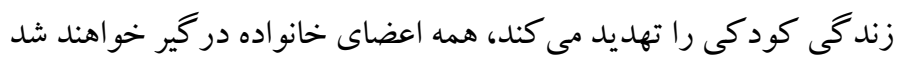

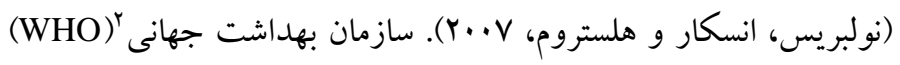

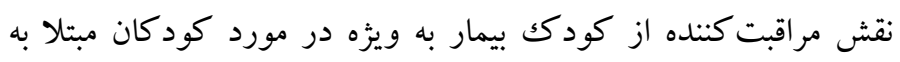

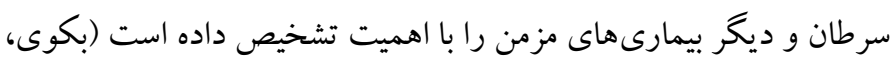

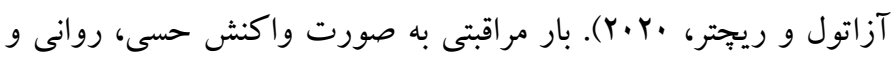

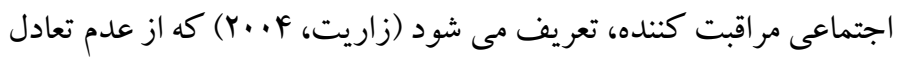
بين نيازهاى مر اقبتى با ديكر وظايف مراقب ناشى مى شود، اين عدم تعادل

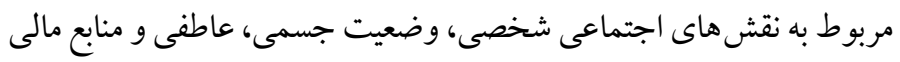

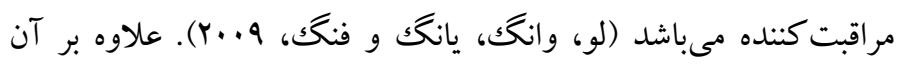
شناسايى به موقع بار مراقبتى در مراقبين، نقش تعيين كنندهاى در ارتقاى

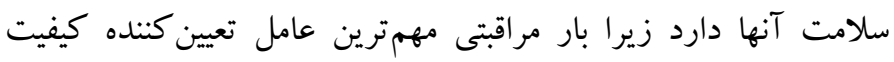
زندكى مراقبين معرفى مىشود. از طرفى ارتباط مستقيم و معنىدارى بين

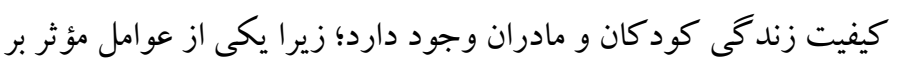

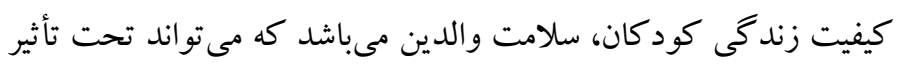

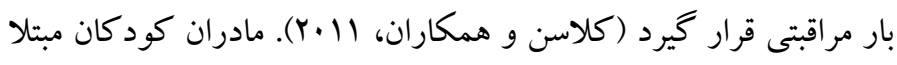

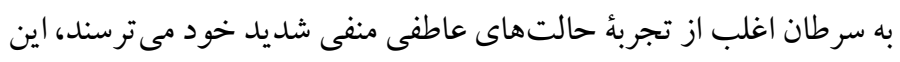
ترس قابل دركك است، زيرا حالتهاى عاطفى منفى آنها اغلب خيلى شديد است، اكرجه تلاش آنها براى اجتناب از اين حالتها، معمولاً نتايج

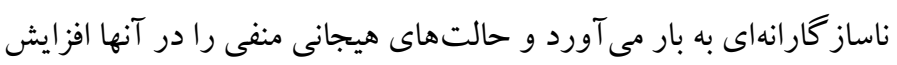

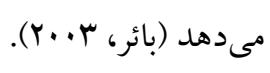

هيجانها" به دلايل مختلف از جمله كاركردهاى تكاملى، اجتماعى ارتباطى، تصميم گيرى، نقش تأثير گذار آنها در سلامت و و بيمارى مورد توجه و مطالعه محققان روانشناسى بوده است. هيجانها، باسخ هاى جند بعدى متشكل از مؤلفهاى تجربى، رفتارى و فيزيولوزيكك هستند (بشارت

3. Emotions
بر اساس تخمين هاى سازمان بهداشت جهانى تا سال Y ب. ب به طور كلى در

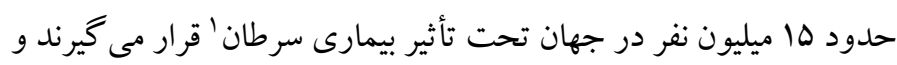

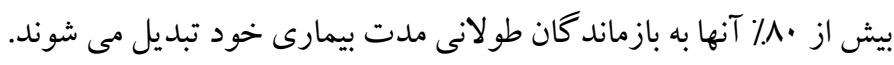

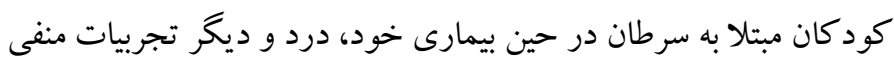

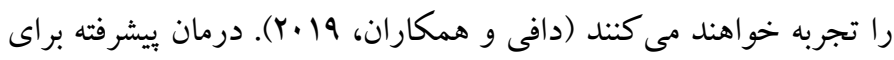
سرطان كودكك اغلب با دو تا سه سال بسترى شدن مكرر و طولانى همراه

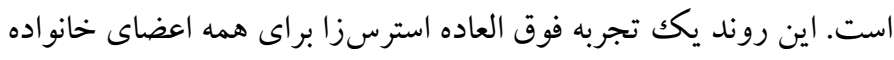

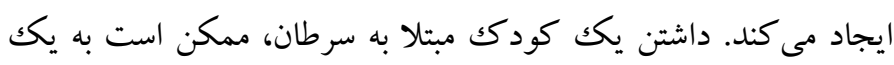

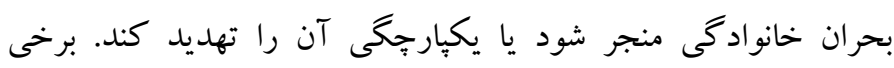

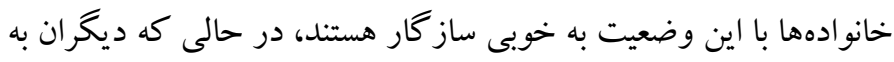
شدت مشكلات جدى را تجربه مى كنند. به عنوان مثال، طلاق و اختلال

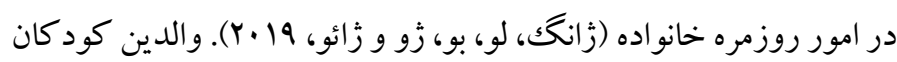

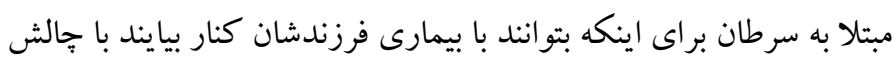

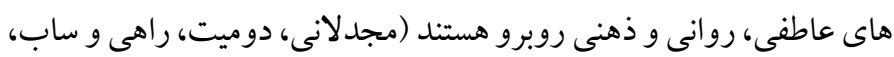
$(Y \cdot) \Lambda$ در گيرى خانو ادگى و اختلالات روانشناختى مادران مى تواند منجر به رفتار

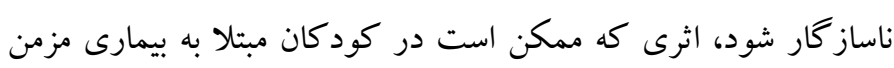

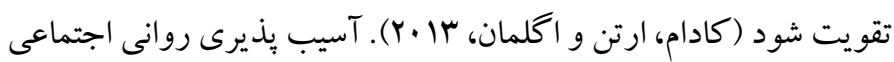

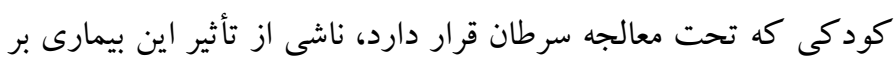

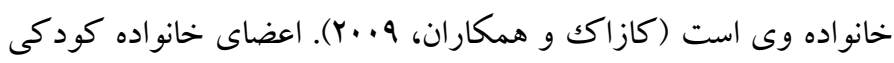

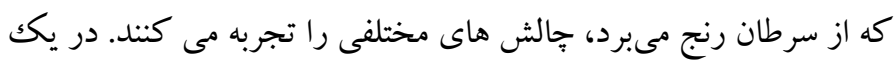

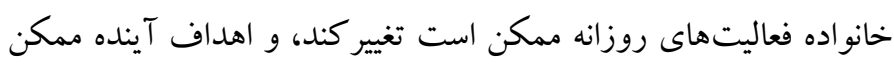

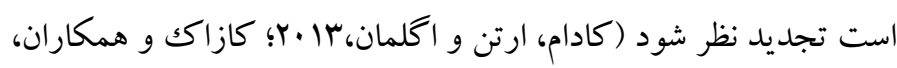

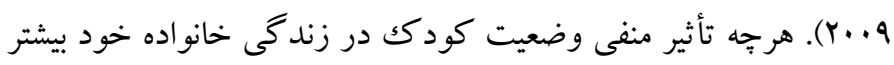

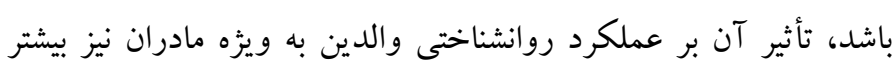

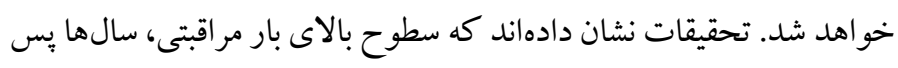
از بايان درمان باقى مى ماند. همجنين علائم بريشانى روانى، از قبيل علائم بـاني

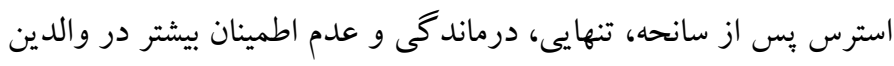

1. Cancer

2. World Health Organization(WHO) 
محتو اى مقصر دانستن و سرزنش ديخران به خاطر آنجه اتفاق افتاده است)،

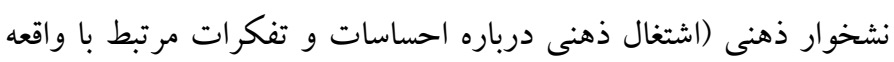

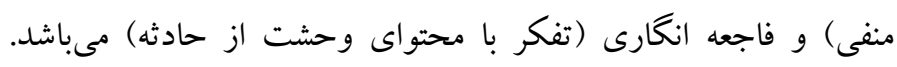

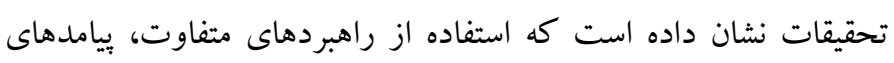

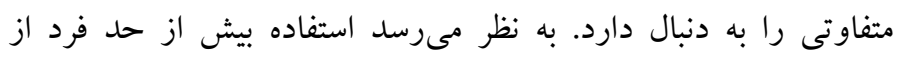

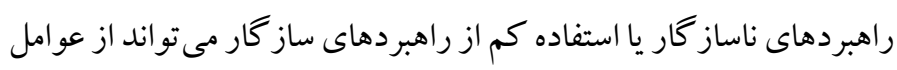
زيربنايى مشكلات هيجانى باشد. استراتزىهاى تنظيم هيجان ناساز گار خطر آناز

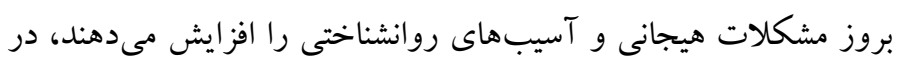
حالى كه راهبردهاى ساز گارانه به عنوان فاكتور محافظتى عمل مى كنيد

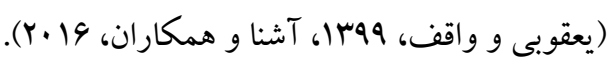

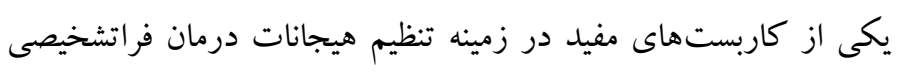
يكيارجه' است كه در باسخ به محدوديت درمان هاى شناختى - رفتارى

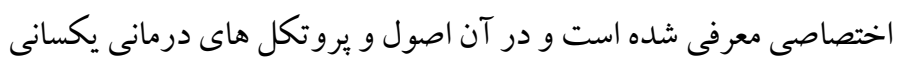

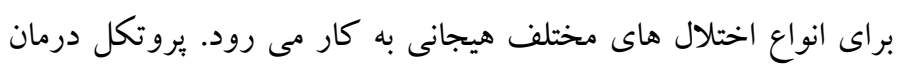

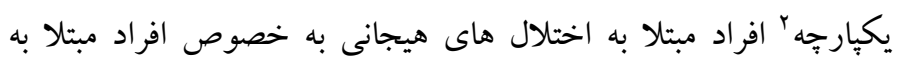
اختلالهاى خلقى و اضطرابى طراحى شده ريشه در سنت شناختى رفتارى دارد و يكك درمان شناختى - رفتارى فراتشخيصى متمر كز بر هيجان است.

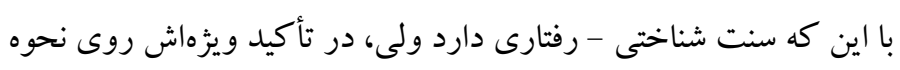

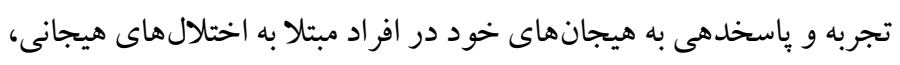

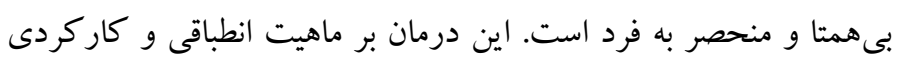

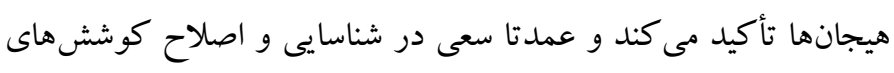
غيرانطباقى براى تنظيم تجارب هيجانى دارد و به واسطه آن بردازش

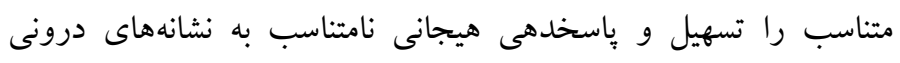

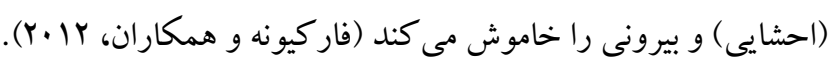
منطق شكل گيرى درمانهاى فراتشخيصى مبتنى بر مفاهيم نظرى و نتايج تجربى در خصوص وجود عوامل مشتركك بين اختلالهاى هيجانى بوده

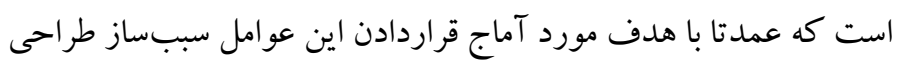

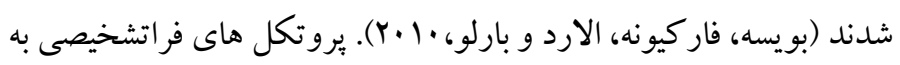

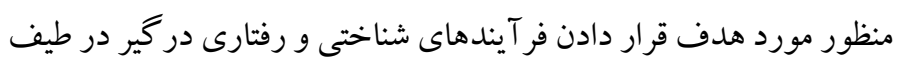

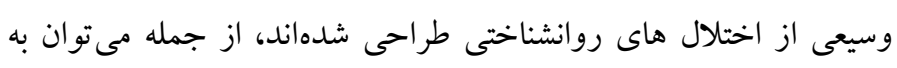

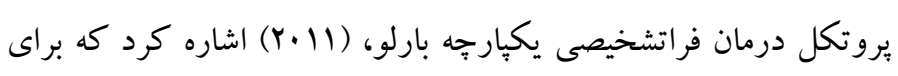

2. Unified Protocol
و بزازيان، سوس|). هيجانها در فر آيند روانشناختى انسانها نقش تعيين كنندهاى دارند. توانايى افراد براى تنظيم شناخت، هيجان و اعمال، يكك

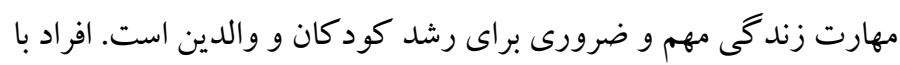

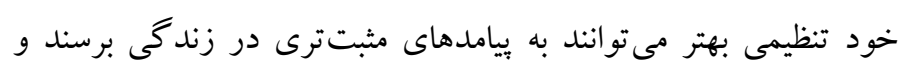
احتمال ايجاد مشكلات ارتباطى و اجتماعى و سلامتروانى كمتر است.

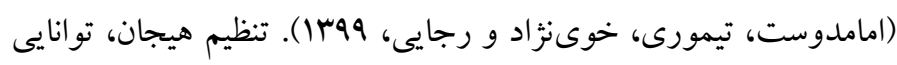
تعديل پارامترهاى يكك تجربه عاطفى است كه شامل دو نوع ياسخ غير

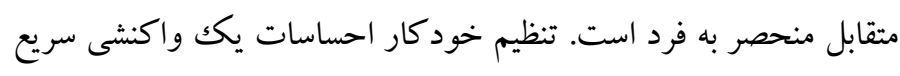
و واكنشى در شروع يكك جالش عاطفى است، در حالى كه تنظيم هيجان آكاهانه يكك واكنش متقابل و طولانى مدت و آشكارتر است. توانايى اجراى تنظيم هيجانى آكاهانه، نگه داشتن ذهن و كنترل آكاهانه، مهارت مهمى است كه در كود كى بديدار مى شود كه عملكرد بعدى در همه زمينه

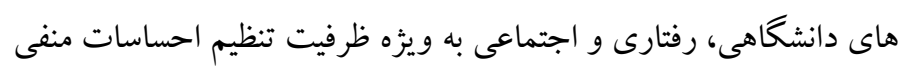

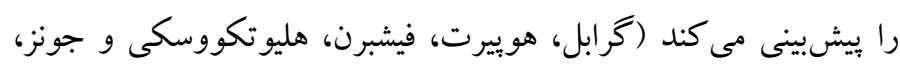

تنظيم هيجان شامل استفاده از راهبردهاى رفتارى و شناختى براى تغيير در مدت زمان و يا شدت تجربه يكك هيجان مىباشد (كرينگك و سولو آن،

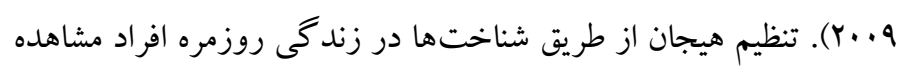

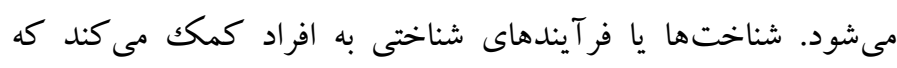

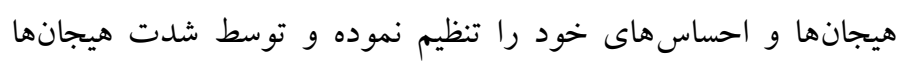

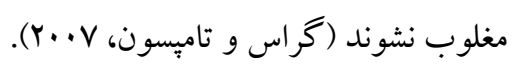

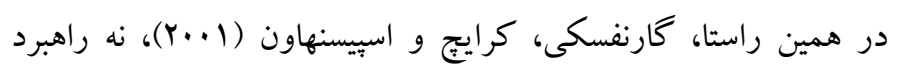

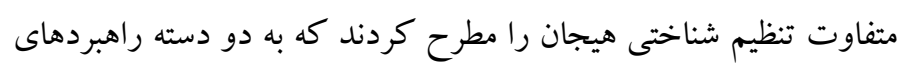

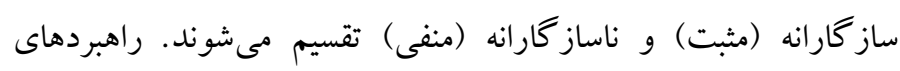

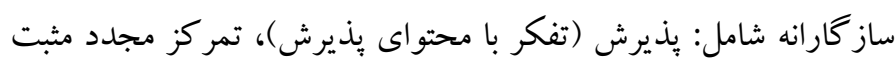

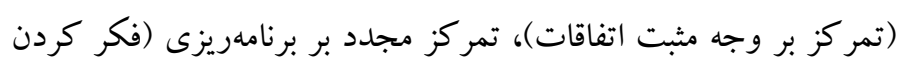

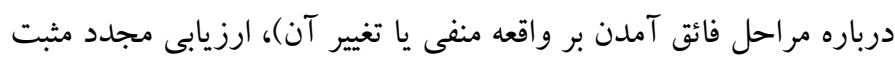

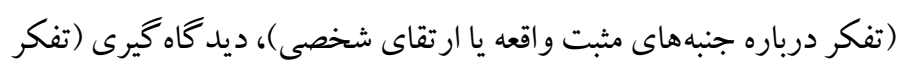
مربوط به كم اهميت بودن واقعه يا تأكيد بر نسبيت آن در مقايسه با ساير

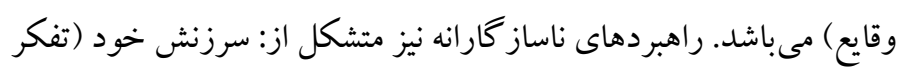
با محتواى مقصر دانستن و سرزنش خود)، سرزنش ديخران (تفكر با

${ }^{1}$. Unified transdiagnostic therapy 
درمان بر كاهش اجتناب تجربى و همجنين كاهش علائم اضطراب و

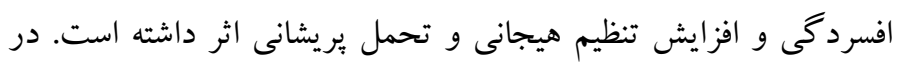

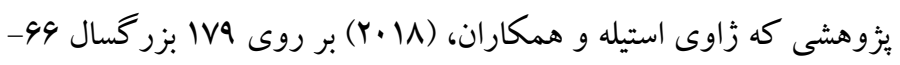

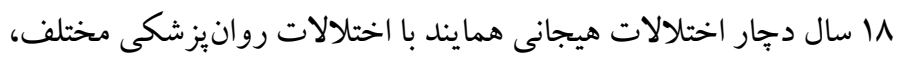

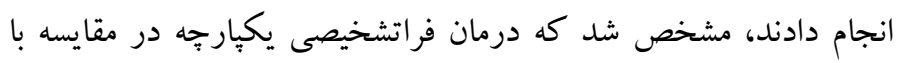
درمانهاى تشخيص محور، باعث كاهش بيشترى در علائم اختلالات

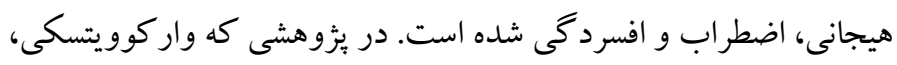

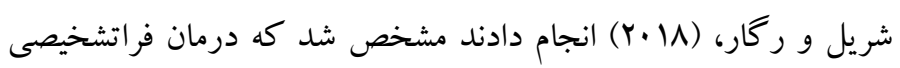

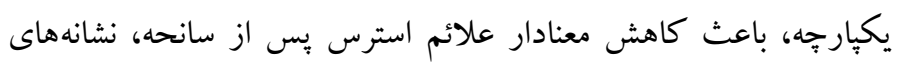

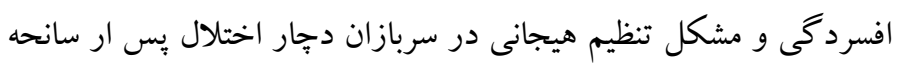

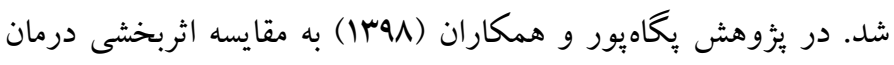
فراتشخيصى و مواجههسازى بازدارى بِاسخ بر اجتناب تجربهاى و تنظيم

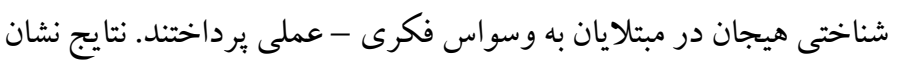

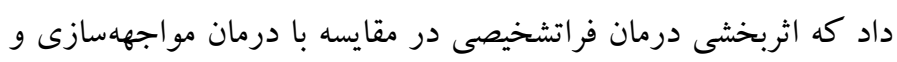

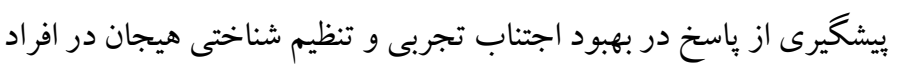

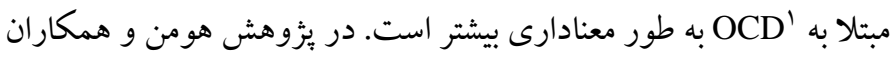

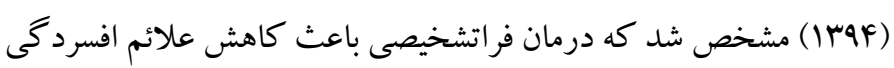
و اضطراب و همجنين افزايش تنظيم شناختى هيجان (افزايش راهبردهاى

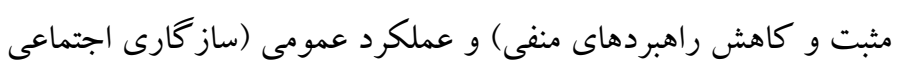

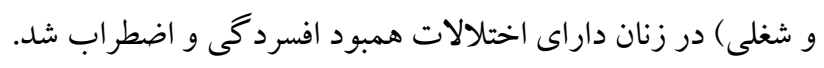
با اين توضيحات مى توان خلا انجام جنين ئزوهش هايى را در مورد مادران داراى كودكك مبتلا به سرطان بيش از ييش احساس كرد. والدين كود كان

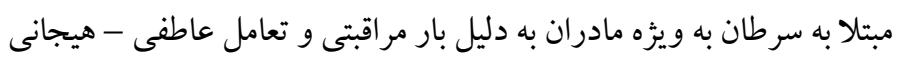

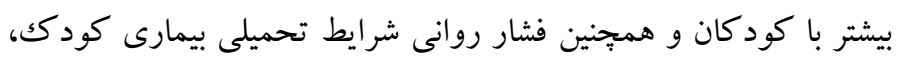

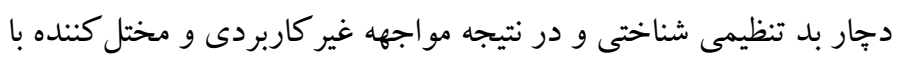
موضوع حاد بيمارى فرزند مىشود كه همين شرايط، والد و كودكى را

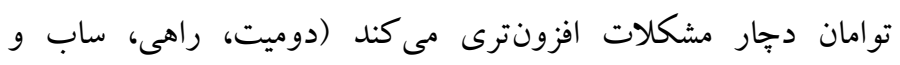

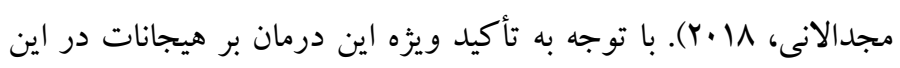

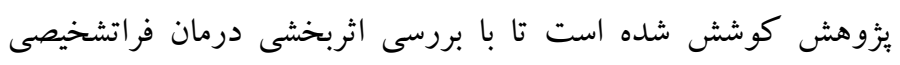

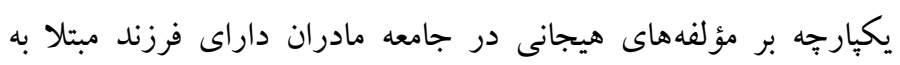

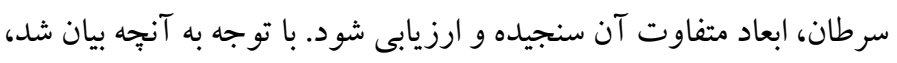

افر اد دار ایى اختلال هاى اضطر ابى و خلقى يكك قطبى با قابليت كاربرديذيرى

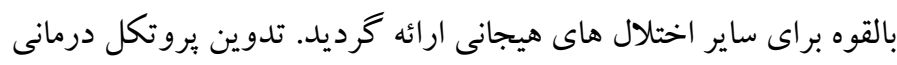

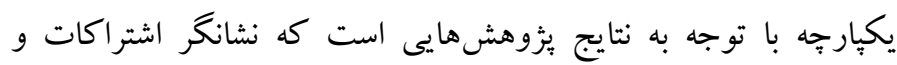

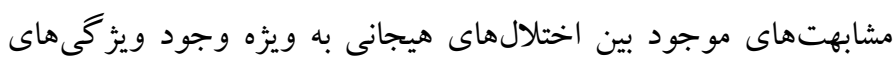
مشترك يا عوامل فراتشخيصى و مشكلات مشتر كك اين اختلالها (مثل:

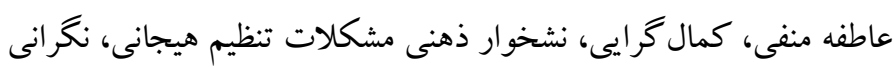

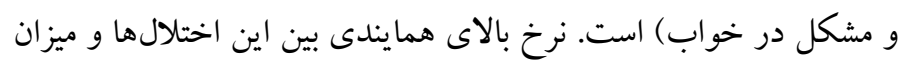

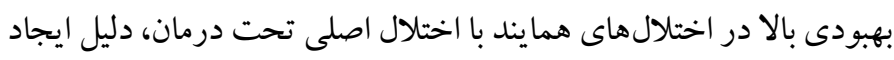

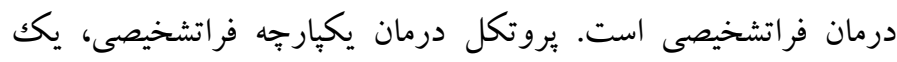

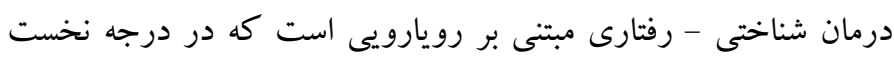

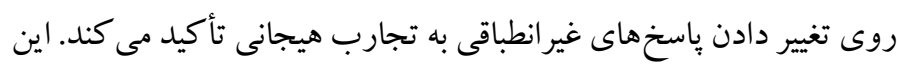

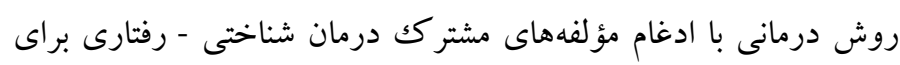

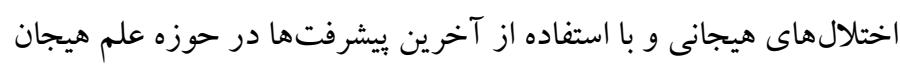

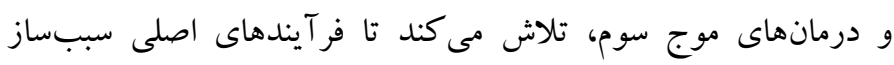
اختلال هاى هيجانى را مورد آماج قرار دهد (بويسه، فار كيونه، الاردو و بارلو،

. (Y. . .

در بثزوهشى كه كاماجو و همكاران (Y.19) بر روى خانوادههاى قربانيان

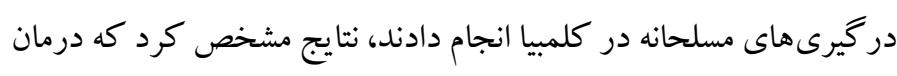

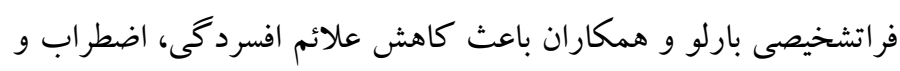
افزايش تنظيم هيجانى و همينطور افزايش كيفيت زندكى در در اين جمعيت مىشود. در فراتحليل و مرور سيستماتيكى كه ساكيريس و برله (Y.19)

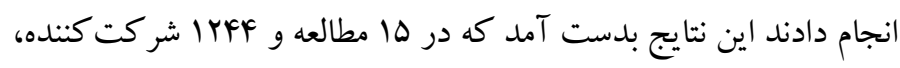
كاهش بسيار معنادار در علائم اضطراب، افسردگى، اختلال اضطراب عمومى، اختلال وسواس اجبارى، اختلال هراس با و بدون آكورافوبيا،

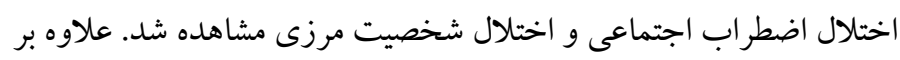
اين، نتايج نشان دهنده افزايش استفاده از راهبردهاى تطبيقى تنظيم هيجانى و كاهش استفاده از استراتزىهاى ناساز گار تنظيم هيجانى بود كه اين نتايج

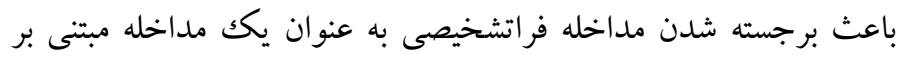

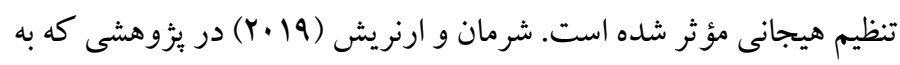

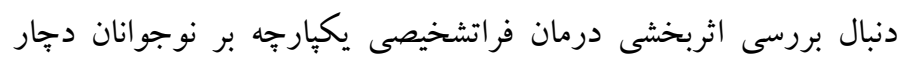

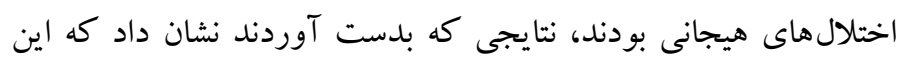


دهند كان محر مانه خو اهد بو د. همجنين به آنها ياد آورى شد كه در صورت

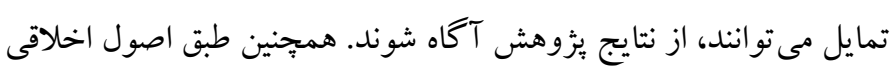

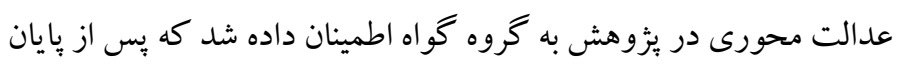

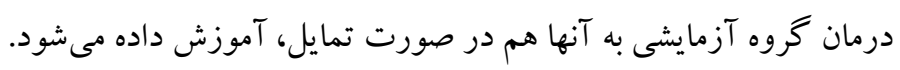

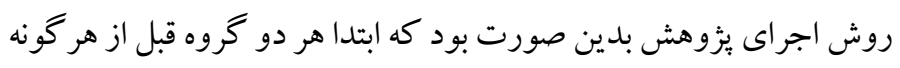
مداخله در شرايط يكسان به برسشنامه راهبردهاى تنظيم شناختى هيجان بردي

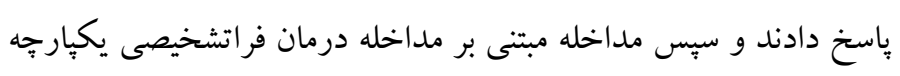

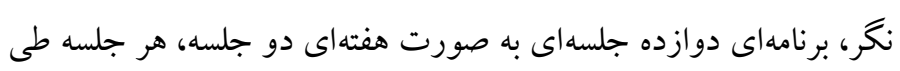

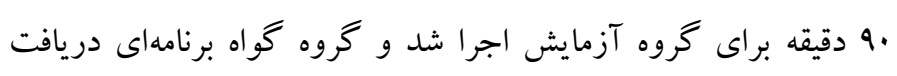
نكردند. و در انتهاى آموزش هم هر دو گروه به برسشنامه هاى بـ بس آزمون

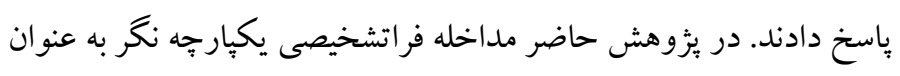
متغيير مستقل و تنظيم شناختى هيجان به عنوان متغير وابسته اعمال شدند. تحليل دادهها با روش تحليل كوواريانس تكك متغيره با استفاده از نرمافزار

SPSS

ب) ب ابز ار

برسشنامه راهبردهاى تنظيم شناختى هيجان' (CERQ) فرم كوتاه (كارنفسكس و كرايج، 9. (Y): يكك برسشنامه خودسنجى است كه نسخه اصلى اين برسشنامه شامل و زير مقياس و داراى وب كويه است. فرم كو تاه

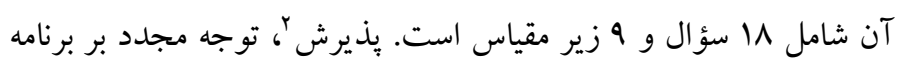

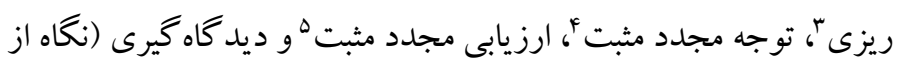

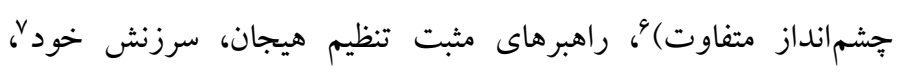

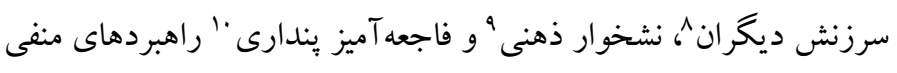

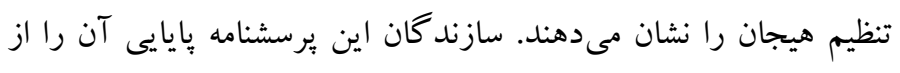

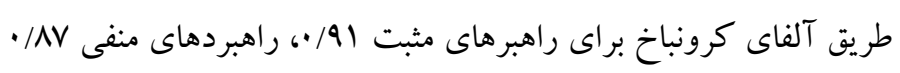

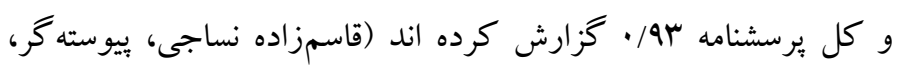

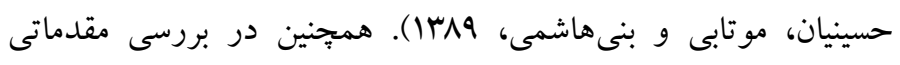

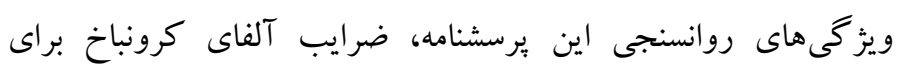

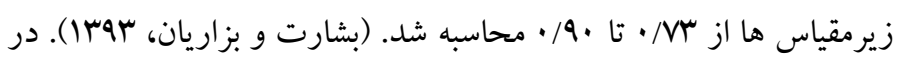

\footnotetext{
6. Putting into Perspective

7. Self- Blam

8. Other-Blam

9 . Focus on Thought/ Rumination

${ }^{10}$. Catastrophizing
}

يزؤهش حاضر در يى ياسخ دادن به اين سؤال است كه آيا درمان

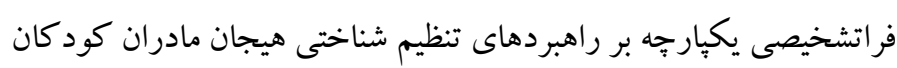

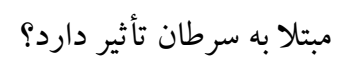

روش الف) طرح يثوهش و شركت كنند كان: يزوهش حاضر از نوع شبه

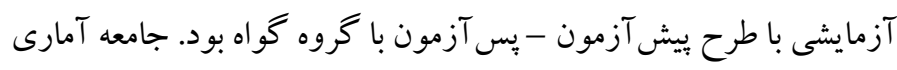

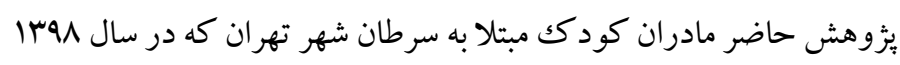
به بيمارستانهاى سطح شهر مراجعه كردهاند، بودند كه براساس سيستم

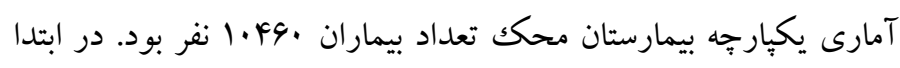

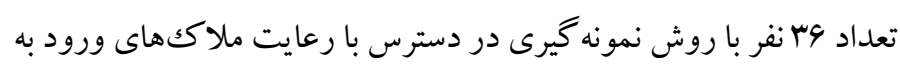

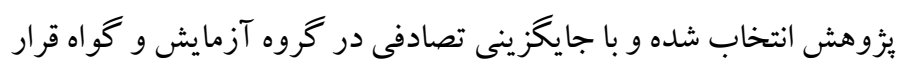

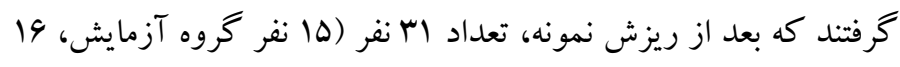

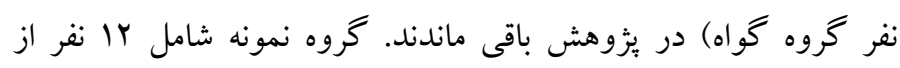

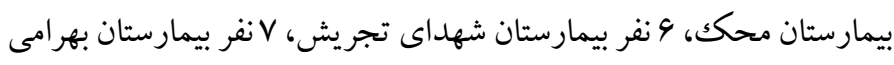

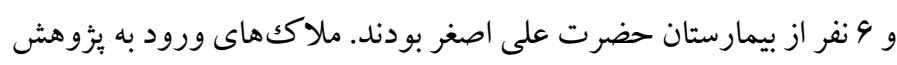

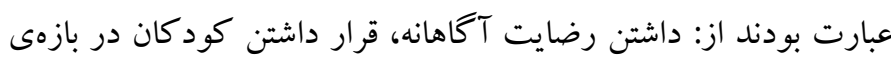
سنى V تا Y I سال و همجِنين تحت درمان بودن كود كان در زمان انجان

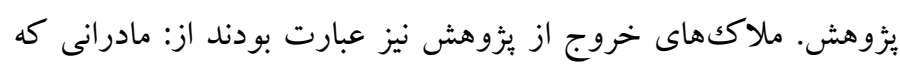
فرزندشان قطع درمان شده يا ترخيص شدهاند، ابتلاى ديخر اعضاى خان خانو اده

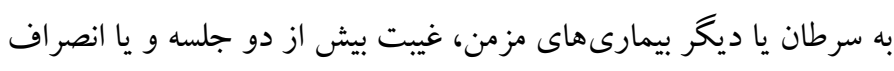

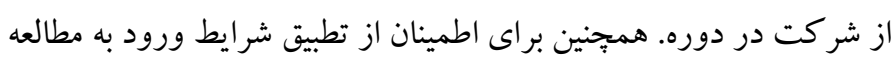

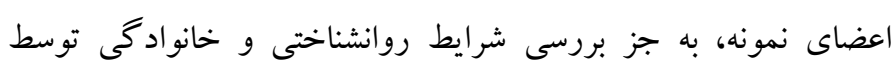

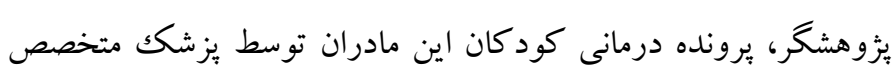
بروسى شد. يزوهش حاضر با كد اخلاق IR.IAU.K.REC.1398.071 در دانشگاه آزاد اسلامى واحد كرج به تصويب رسيده است. همجنين جهت رعايت اصول

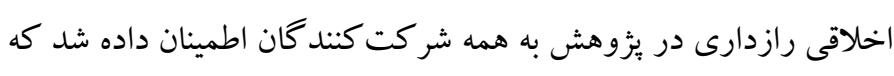

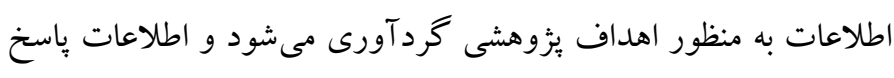

1. Cognitive Emotion Regulation Questionnaire(CERQ)

2. Acceptance

3. Refocus of Planing

${ }^{4}$. Positive Refocusing

5. Positive Reappraisal 
بالاتر نشان دهنده استفاده بيشتر فرد از آن راهبرد شناختى محسوب مى شود. راهبرد شناختى تنظيم هيجان در پرسشنامه تنظيم شناختى هيجان به دسته كلى راهبردهاى انطباقى (سازش يافته) و راهبردهاى غير انطباقى (سازش نايافته) تقسيم مىشوند. در جدول ا خلاصه جلسات درمانى فر اتشخيصى

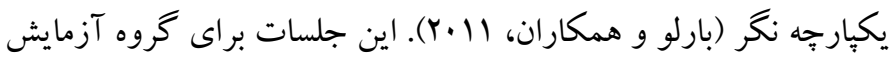
اجرا شده است.
اين بثروهش از فرم كو تاه برسشنامه تنظيم شناختى هيجان استفاده شده است. ياسخهاى اين يرسشنامه در يكك ييوستار ينج درجهاى (هميشه، اغلب اوقات، معمولاً، گاهى، هر گز) جمع آورى مىشود. براى نمره خذارى

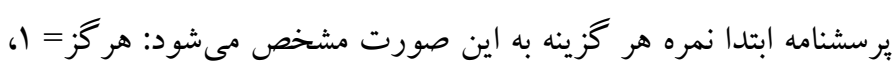

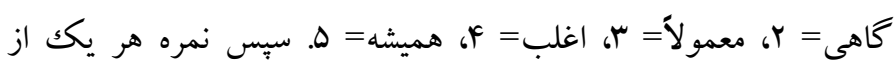
زيرمقياس هاى q كانه با جمع نمرههاى دو مادّه هر زيرمقياس محاسبه مى شود حداقل و حداكثر نمره در هر زيرمقياس به ترتيب Y و · ا است و نمره

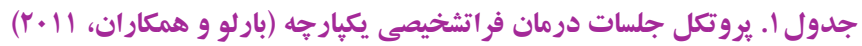

\begin{tabular}{|c|c|c|c|}
\hline تكاليف & و اهدح محتواف جلسات رفتارى آنها & 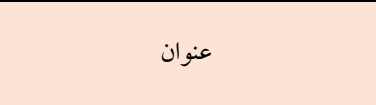 & 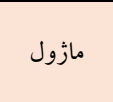 \\
\hline مشخص كردن مز ايا و معايب تغيير، اهداف عينى و گامهاى درمان & 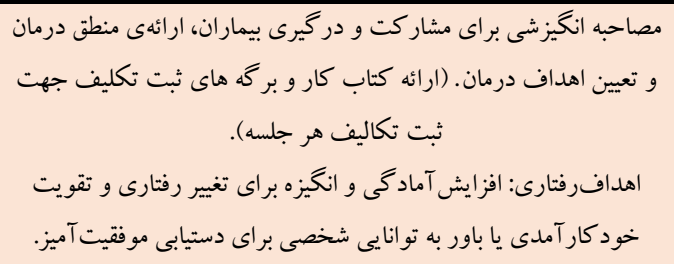 & افزايش انخيزه براى شركت در درمان & مازول 1 \\
\hline 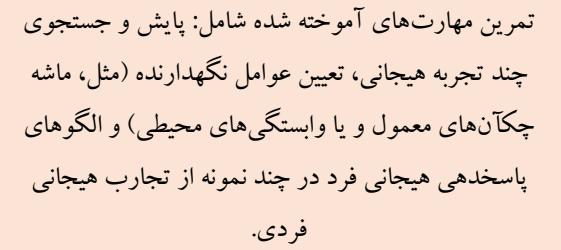 & 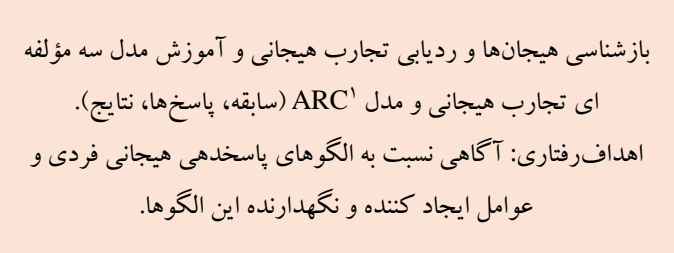 & آموزش روانشناختى و جستجوى تجربه & 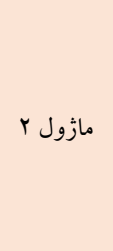 \\
\hline 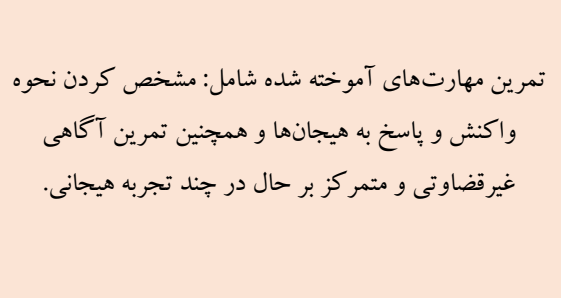 & 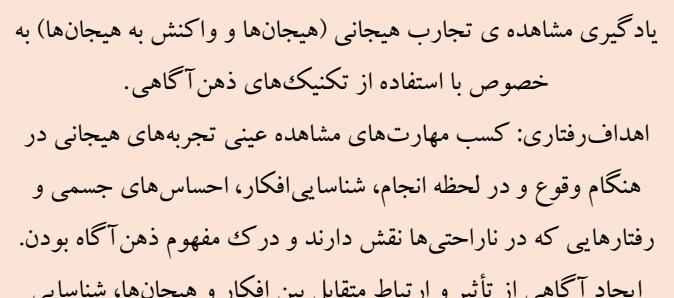 & آموزش آكاهى هيجانى & مازول r ماو \\
\hline 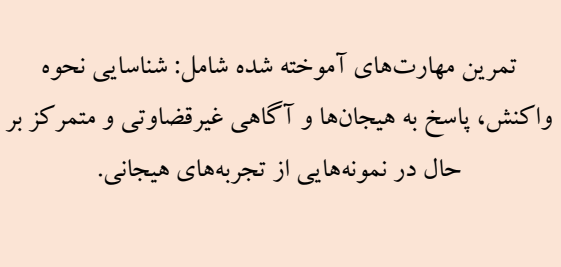 & 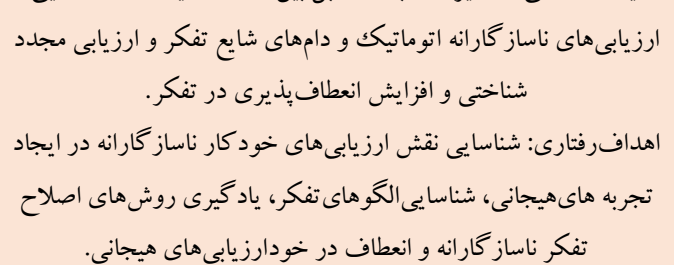 & بازارزيابى و ارزيابى مجدد شناختى & 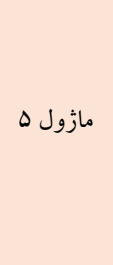 \\
\hline 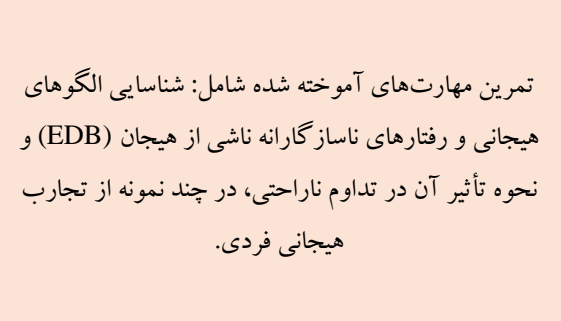 & 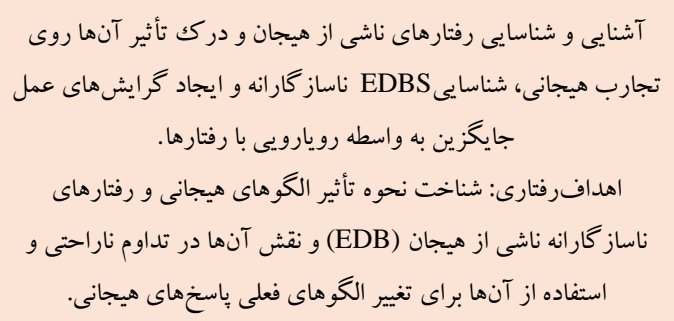 & 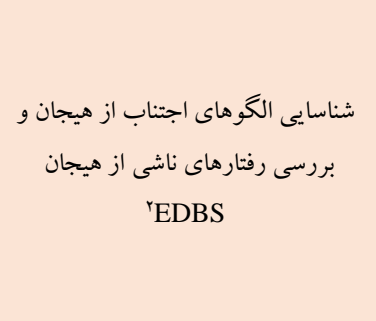 & $\begin{array}{c}\text { مازول } 9 \text { و } \\
V\end{array}$ \\
\hline
\end{tabular}

2. Emotion Driven Behaviors

1. Anticedents,Responses, Consequences 


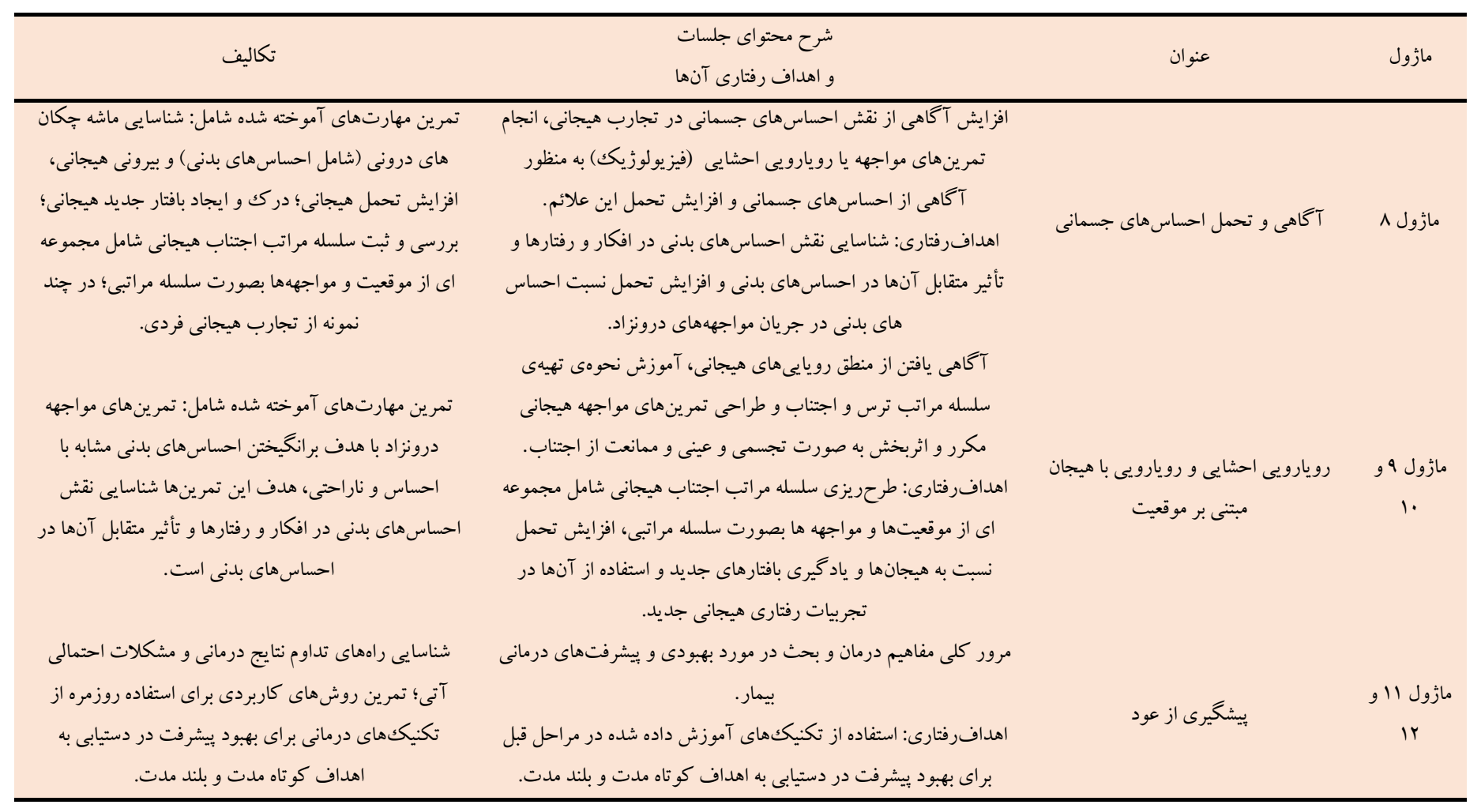

كروه آزمايش ميانگين مؤلفه يذيرش، تمر كز مجدد مثبت، تمر كز مجدد بر

يافته ها

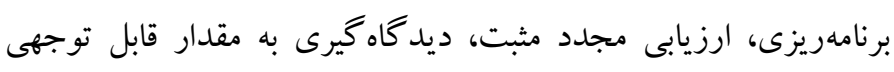
اطلاعات جمعيت شناختى شركت كنند گان در يزوهش حاضر شامل موارد

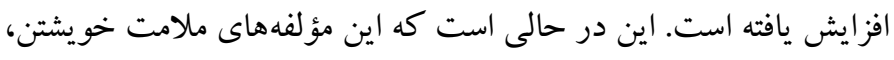

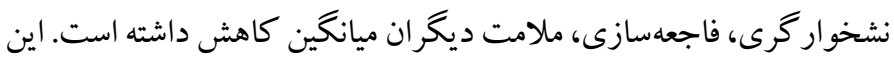

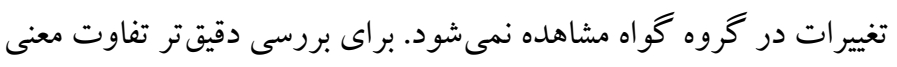

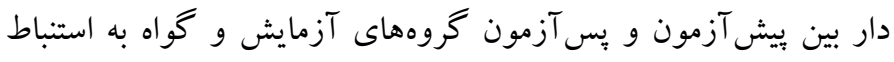

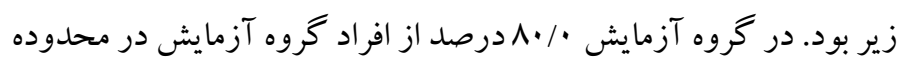

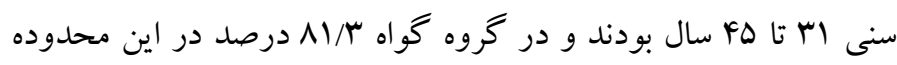

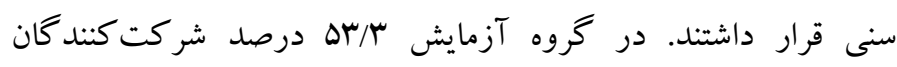

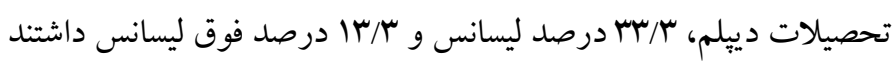
آمارى خواهيم يرداخت.

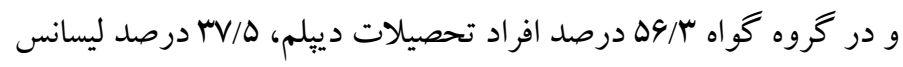
و 9/T درصد فوق ليسانس داشتند. در خروه آزمايش 94/V درصد متأهل و و

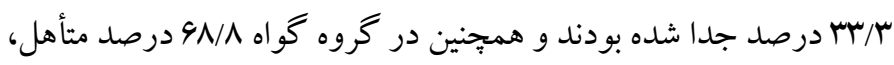

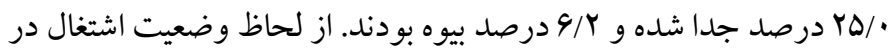

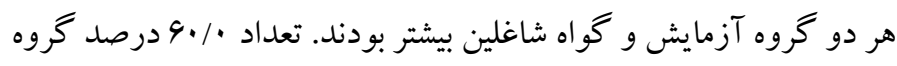

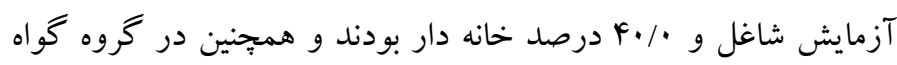

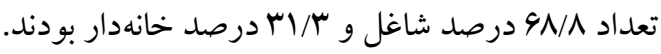
در جدول r آمار توصيفى متغير اصلى يزٔوهش (مؤلفهاى تنظيم شناختى

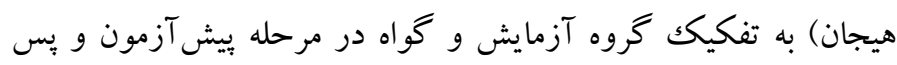

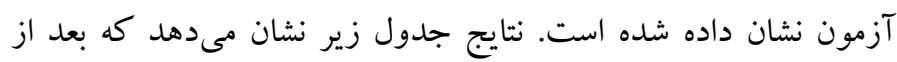
مداخله فراتشخيصى يكيارجهن 


\begin{tabular}{|c|c|c|c|c|c|}
\hline انحراف معيار & ميانگين & فراوانى & مرحله & كروه & مؤلفه \\
\hline$|/| 4 \mid$ & V/Ir & 10 & يَيش آزمون & \multirow{2}{*}{ آ آزمايش } & \multirow{4}{*}{ ملامت خويشتن } \\
\hline$|/ 4|$ & $r / l r$ & 10 & يس آزمون & & \\
\hline $1 / 94$ & $9 / \mu 1$ & 19 & بيش آزمون & \multirow{2}{*}{ كواه } & \\
\hline $1 / M$ & $9 / 9 F$ & 19 & يس آزمون & & \\
\hline $1 / r \Delta$ & $r / A V$ & 10 & ي بيش آزمون & \multirow{2}{*}{ آزمايش } & \multirow{4}{*}{ ل يذيرش } \\
\hline $1 / 9 V$ & $9 / 1$. & 10 & يس آزمون & & \\
\hline $1 / 9 \mathrm{~V}$ & $F / \mu_{\Lambda}$ & 19 & ي بيش آزمون & \multirow{2}{*}{ كواه } & \\
\hline $1 / 94$ & $r / 99$ & 19 & يس آزمون & & \\
\hline $1 / \Delta \Lambda$ & $V / r V$ & 10 & ي بيش آزمون & \multirow{2}{*}{ آزمايش } & \multirow{4}{*}{ نشخوارگرى } \\
\hline $1 / V V$ & $r / l r$ & 10 & يس آزمون & & \\
\hline $1 / 94$ & $9 / 11$ & 19 & ي ي بيش آزمون & \multirow{2}{*}{ كواه } & \\
\hline$r / \cdot \Lambda$ & $9 / 9 F$ & 19 & يس آزمون & & \\
\hline $1 / \Delta$ & $\varphi / 9$. & 10 & يبش آزمون & \multirow{2}{*}{ آزمايش } & \multirow{4}{*}{ تمركز مجدد مثبت } \\
\hline $1 / N r$ & V/Ir & 10 & يس آزمون & & \\
\hline $1 / \& \Delta$ & $f / I r$ & 19 & ي بيش آزمون & \multirow{2}{*}{ كواه } & \\
\hline$|/ 4|$ & $F / M V$ & 19 & يس بـ آزمون & & \\
\hline $1 / \Delta \Lambda$ & $r / \cdot v$ & 10 & ي يش آزمون & \multirow{2}{*}{ آازمايش } & \multirow{4}{*}{ تمركز مجدد بر برنامهريزى } \\
\hline$r / 19$ & $V / F$. & 10 & يس آزمون & & \\
\hline $1 / N r$ & $F / V \Delta$ & 19 & ي يش آزمون & \multirow[t]{2}{*}{ كواه } & \\
\hline $1 / \Delta r$ & $F / Y \Delta$ & 19 & يس آزمون & & \\
\hline 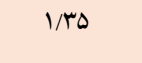 & $\mathrm{r} / 9 \mathrm{~V}$ & 10 & ي بيش آزمون & \multirow{2}{*}{ آزمايش } & \multirow{4}{*}{ ارزيابى مجدد مثبت } \\
\hline $1 / 9$. & V/Ir & 10 & 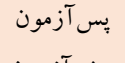 & & \\
\hline I/VY & $F / \wedge)$ & 19 & ي ي بش آزمون & \multirow[t]{2}{*}{ كواه } & \\
\hline I/VD & $F / F F$ & 19 & 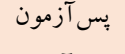 & & \\
\hline $1 / \cdot 1$ & $F / r$. & 10 & ييش آزمون & \multirow[t]{2}{*}{ آزمايش } & \multirow{4}{*}{ ديدگاهيرى } \\
\hline $1 / N 1$ & $\mathrm{~V} / \cdot \mathrm{V}$ & 10 & يس آزمون & & \\
\hline $1 / v$. & $4 / 99$ & 19 & ي ي & \multirow[t]{2}{*}{ كواه } & \\
\hline $1 / F F$ & $4 / .9$ & 19 & 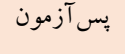 & & \\
\hline $1 / 91$ & $\mathrm{~V} / \mathrm{A} \cdot$ & 10 & ي يِش آزمون & \multirow{2}{*}{ آزمايش } & \multirow{4}{*}{ فاجعهسازى } \\
\hline $1 / M$ & $f / r$ & 10 & 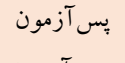 & & \\
\hline $1 / 49$ & $V / 99$ & 19 & ي بيش آزمون & \multirow[t]{2}{*}{ كواه } & \\
\hline $1 / V 9$ & $\wedge / \cdot \cdot$ & 19 & 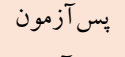 & & \\
\hline $1 / \Delta V$ & $\Lambda / r$. & 10 & ي ي يش آزمون & \multirow{2}{*}{ آزمايش } & \multirow{4}{*}{ ملامت ديخران } \\
\hline $1 / M$ & $F / \Delta r$ & 10 & מֶ آزمون & & \\
\hline 1/99 & $V / \mu 1$ & 19 & ي ي آزمون & \multirow[t]{2}{*}{ كواه } & \\
\hline $1 / \wedge 1$ & $1 / \cdot 9$ & 19 & پِ آزمون & & \\
\hline
\end{tabular}

جهت بررسى اين فرضيه و كنترل تفاوتهاى آزمودنىها در يیش آزمون از بررسى نرمال بودن دادهها، آزمون لون براى سنجش برابرى واريانسها و

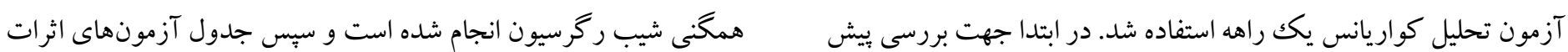

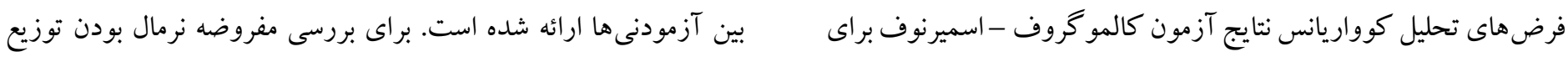


صورت جداكانه انجام گرفت كه نتايج حاصل در جدول ب مشاهده مى شود. نتايج نشان مىدهد كه مقدار F محاسبه شده براى خرده مقياس هاى

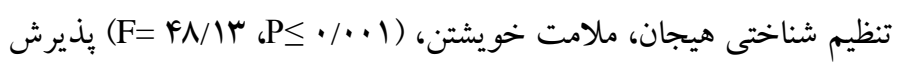

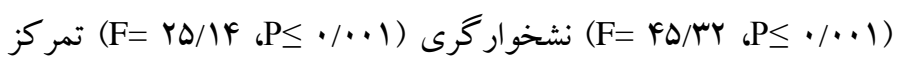

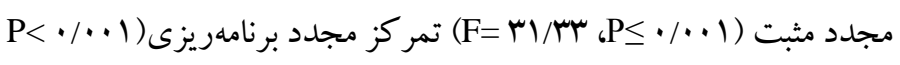

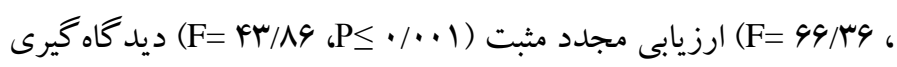
فاجعdسازى (F= MF/94 $\left.{ }_{6} \mathrm{P} \leq \cdot / \cdots\right)$

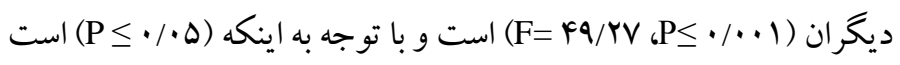
در نتيجه مقدار F محاسبه شده معنادار است كه بيانگر تفاوت اثر شرايط آزمايش بر متغير وابسته (مؤلفهاى مثبت و منفى تنظيم شناختى هيجان)
نمرات متغيرها از آزمون كالمو گروف - اسميرنوف استفاده شده است. به دليل آن كه سطح معنىدارى در آزمون ذكر شده از هـ/ • بزركت تر بود مىتوان نتيجه كرفت كه توزيع نمرات متغيرهاى وابسته نرمال است

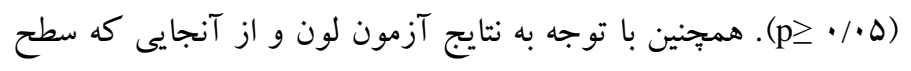

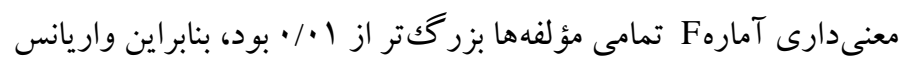
خطاى دو كروه با هم برابر بوده و تفاوتى بين آنها مشاهده نمىشود. همجنين مقدار F تعامل متغير همٍراش براى متغيرها معنادار نيست

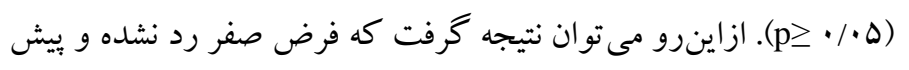
فرض همخنى شيب رگر سيون رعايت شده است. براى يى بردن به اين نكته كه از لحاظ كدام متغير يا متغيرها بين دو گروه تفاوت وجود دارد، تحليل

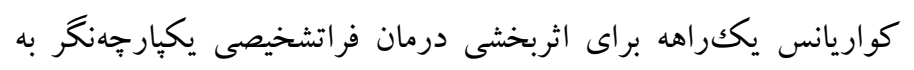

است.

جدول ". آزمون تحليل واريانس يككراهه براى متغير راهبردهاى تنظيم شناختى هيجان

\begin{tabular}{|c|c|c|c|c|c|c|c|c|}
\hline توان آزمون & اتا & سطح معنادارى & F & ميانگين مجذورات & درج آزادى & مجموع مجذورات & شاخص منبع تغييرات & متغيير \\
\hline$\cdot / 99$ & $|4|$ & $\cdot / \cdot 1$ & 19/תr & $r Y / q \Delta$ & 1 & $r Y / Q \Delta$ & بيش آزمون & \multirow{4}{*}{ ملامت خويشتن } \\
\hline $1 / \cdot$ & .194 & $\cdot / \cdot .1$ & FN/IT & $\Lambda r / \cdot r$ & 1 & $\Lambda r / \cdot r$ & آزمايش & \\
\hline- & - & - & - & $1 / v$ & rA & FV/VY & خطا & \\
\hline- & - & - & - & - & r & $\| 1 \cdot v / .$. & كل & \\
\hline$\cdot / 94$ & •//q &.$/ \cdot 1$ & IN/YF & $\mathrm{rV} / \cdot 1$ & 1 & $\mathrm{rV} / \cdot 1$ & ييش آزمون & \multirow{4}{*}{ ل يذيرش } \\
\hline $1 /$. & $\cdot / 94$ &.$/ . \cdot 1$ & $F \Delta / T Y$ & $91 / 91$ & 1 & $91 / 91$ & آزمايش & \\
\hline- & - & - & - & $r / \cdot r$ & rA & $\Delta G / A r$ & خطا & \\
\hline- & - & - & - & - & r & $\mid \cdots \Delta / \cdots$ & كل & \\
\hline$\cdot / 19$ & $\cdot / 49$ & $\% F$ & $9 / 91$ & YN/FY & 1 & YN/Fr & يِيش آزمون & \multirow{4}{*}{ نشخوارگرى } \\
\hline $1 /$. & $\cdot / q v$ & $\cdot / \cdot .1$ & $r \Delta / I F$ & $V Y / .4$ & 1 & $V Y / \cdot 9$ & آزمايش & \\
\hline- & - & - & - & Y/AV & rی & $\Lambda \cdot / r \Delta$ & خطا & \\
\hline- & - & - & - & - & r & $\| r \Delta /$. & كل & \\
\hline $1 / \cdot$ & $\cdot / \& \Delta$ & $\cdot / \cdot 1$ & rY/VF & $r r / \cdot r$ & 1 & $r r / \cdot r$ & ييش آزمون & \multirow{4}{*}{ تمر كز مجدد مثبت } \\
\hline $1 / \cdot \cdot$ & $\cdot / \Delta r$ &.$/ \cdots 1$ & r/M & $F F / / F$ & 1 & $F F / I F$ & آزمايش & \\
\hline- & - & - & - & $|/ 4|$ & rA & $r q / F \Delta$ & خطا & \\
\hline- & - & - & - & - & r & $\| \not+1 /$. & كل & \\
\hline $1 / \cdot$ & $\cdot / \Delta V$ &.$/ . .1$ & rG/NG & $\Delta V / I V$ & 1 & $\Delta V / I V$ & يِيش آزمون & \multirow{4}{*}{ تمركز مجدد بر برنامهريزى } \\
\hline $1 / \cdot$ & $\cdot / v$ &.$/ \cdot 1$ & $99 / 149$ & $1 . r / q F$ & 1 & $1 . r / q F$ & آزمايش & \\
\hline- & - & - & - & $1 / \Delta \Delta$ & rı & $F r / F r$ & خطا & \\
\hline- & - & - & - & - & m & $|r| 1 / \cdot$ & كل & \\
\hline.$/ 91$ & $\cdot / r V$ &.$/ . .1$ & $19 / 0 \mathrm{r}$ & $r \cdot / r$. & 1 & $r \cdot / r \cdot$ & ي بيش آزمون & \multirow{4}{*}{ ارزيابى مجدد مثبت } \\
\hline $1 / \cdot$ & .191 & $\cdot / \cdot .1$ & $\mathrm{Kr} / \mathrm{AG}$ & $\Lambda \cdot / q V$ & 1 & $\Lambda \cdot / q v$ & آزمايش & \\
\hline- & - & - & - & l/Ar & rA & $\Delta I / T V$ & خطا & \\
\hline- & - & - & - & - & m & $119 \cdot /$. & كل & \\
\hline$\cdot / \Delta r$ & $\cdot / / F$ & $\cdot / \cdot r$ & $F / \mu_{\Lambda}$ & Q/Vr & 1 & $9 / V Y$ & ي بيش آزمون & ديد گاه گيرى \\
\hline
\end{tabular}




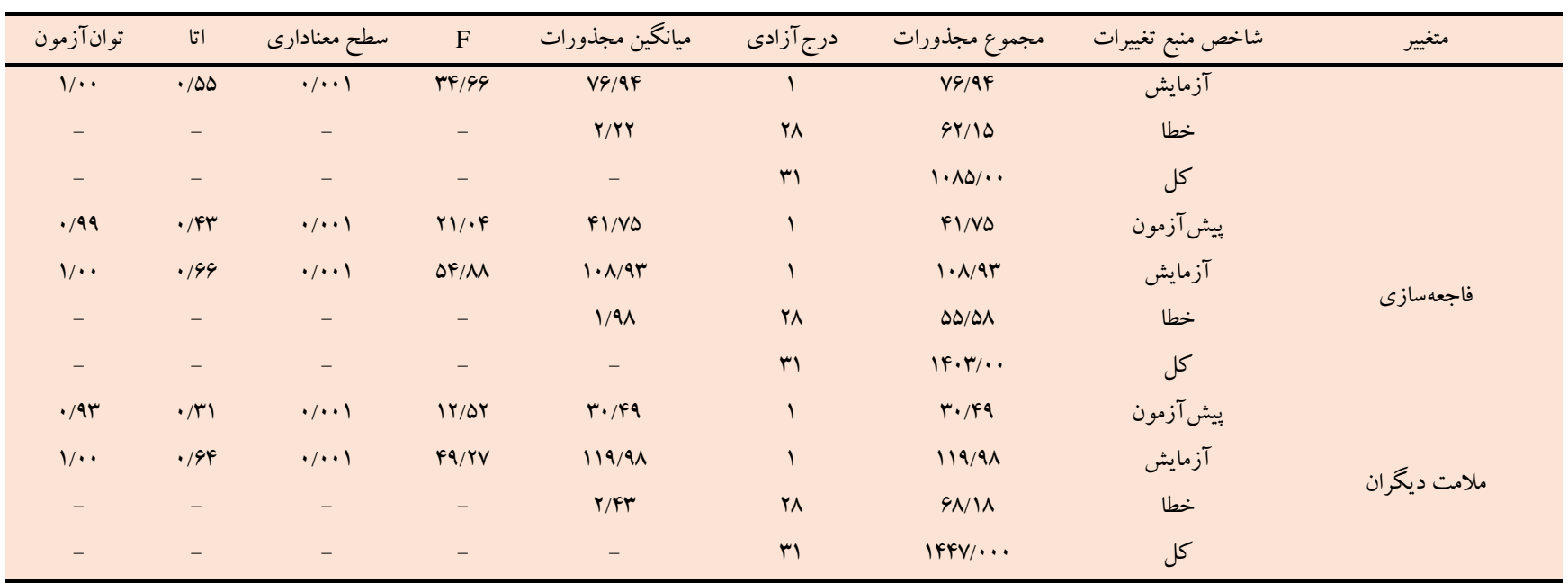

مى تواند به بىثباتى عاطفى - هيجانى، ايجاد اخلال در تنظيم هيجانى، عدم

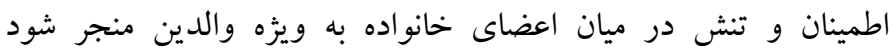

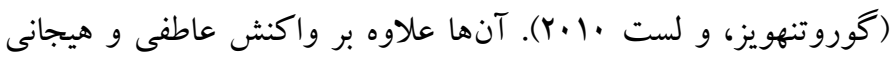

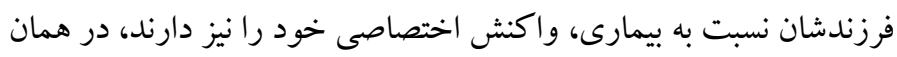

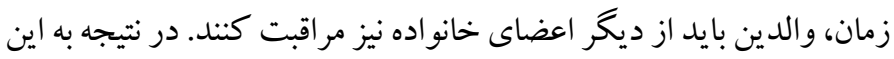

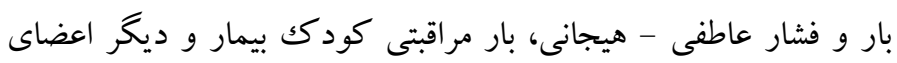

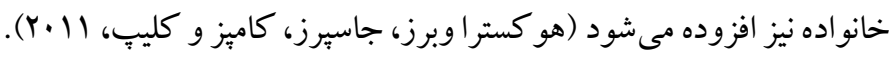
لذا براى كاهش اين تنش و عدم نظم شناختى هيجان، نياز به مداخلههايى

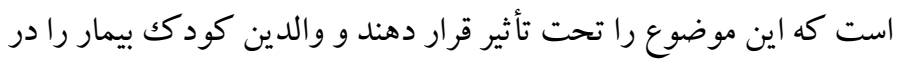
حيطه بازيابى و ترميم روانشناختى مورد درمان و حمايت قرار دهند.

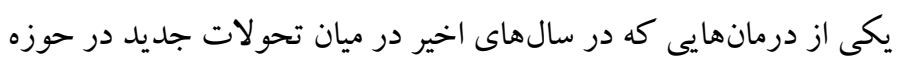
درمانهاى شناختى - رفتارى در مقابل درمانهاى اختصاصى براى اختلال

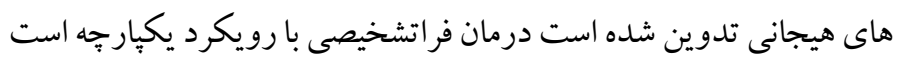

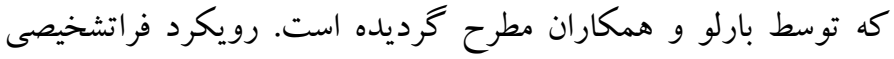
مؤلفههاى زيربنايى آسيب روانى را مورد هدف قرار مىدهد و مىتو اند براى اختلالها و مشكلاتى كه زيربناى يكسان و مشابهى دارند كاربرد

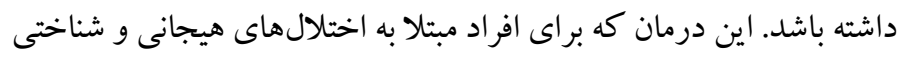

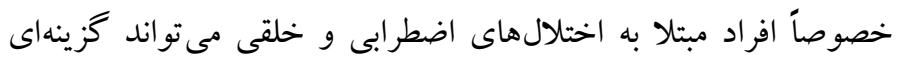

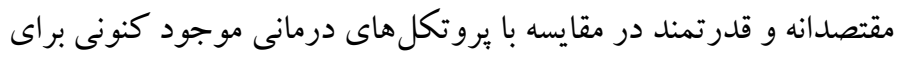

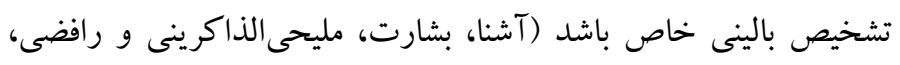

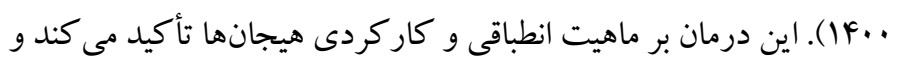
عمدتا سعى در شناسايى و اصلاح كوششهاى غيرانطباقى براى تنظيم
بحث و نتيجه كيرى

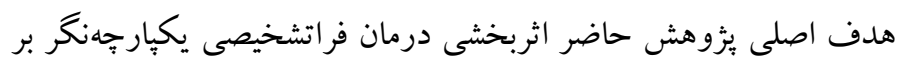

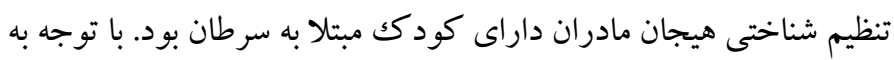

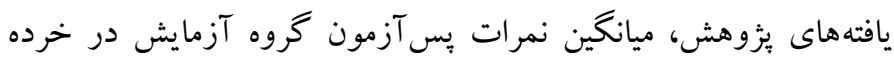

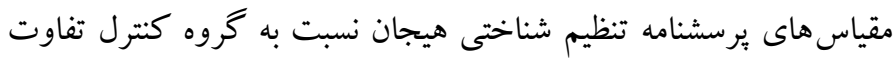

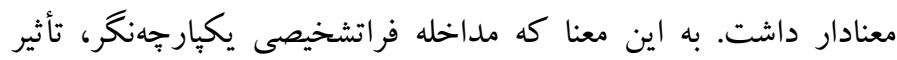
معنادارى بر افزايش استفاده از راهبردهاى سازش يافته و كاهش استفاده از

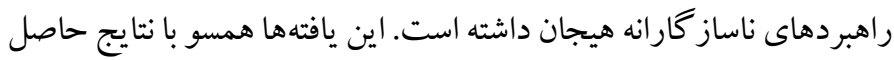

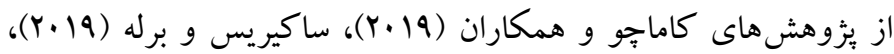

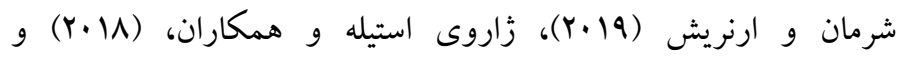

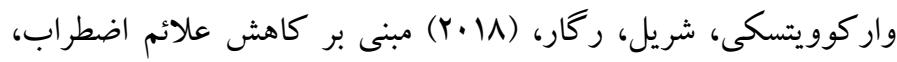
افزايش استفاده از راهبردهاى تطبيقى تنظيم هيجانى و كاهش استفاده از رداز

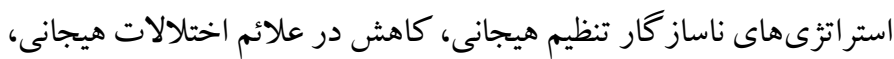
نشانهاي افسردگى و مشكل تنظيم هيجانى همسو است.

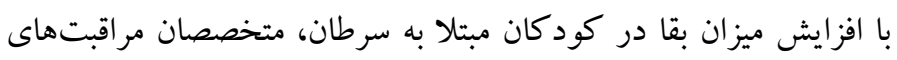
بهداشتى از نقش بسيار مهم والدين در مراقبت اين كود كان آكاه شدهاند. والدين نه تنها نقش خاصى در مراقبت كودكك در حين درمان سرطان

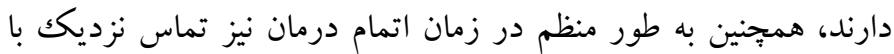

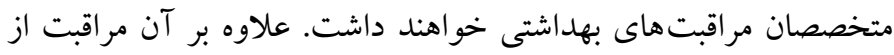
كودك زندگى معمول آنها و همجنين نقشهاى والدينى تغيير مى يابد،

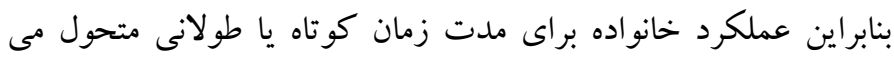

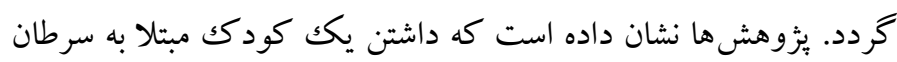


انواعى از ياسخهاى مقابلهاى را به كار ببرند. مهارت خودنخرى مىتواند

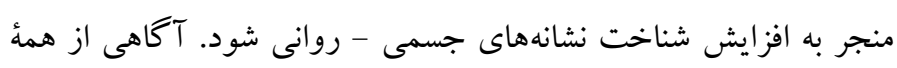

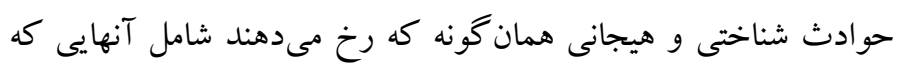

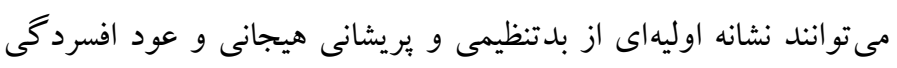

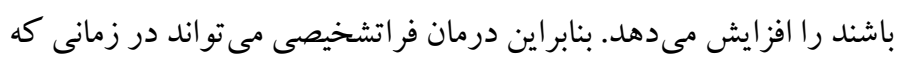

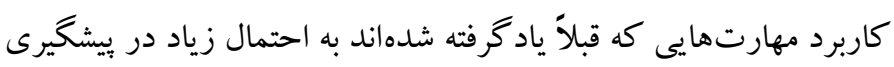

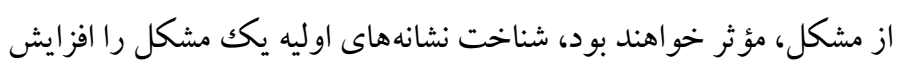

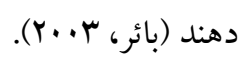

در تبينى ديخر درمان فراتشخيصى مبتى بر تمركز بر لحظه حاضر و حالت

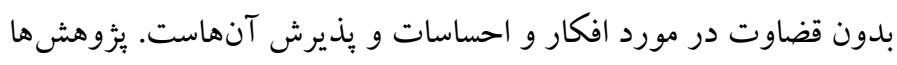
نشان داده است كه تمركززدايى مىتواند سطوح نشخوار افسردهاز را

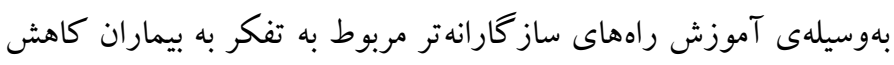
دهد ايدهى اصلى تمر كززدايى مطرح كردن تغييرات اساسى در ديد گاهها،

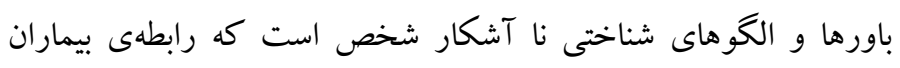

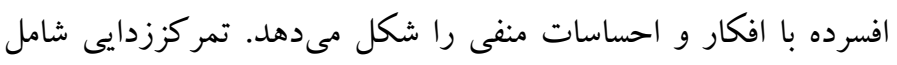

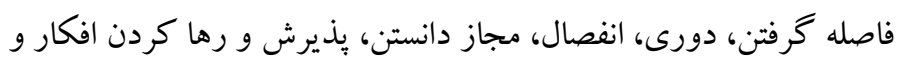
خلقيات منفى است. مادران در برنامهى فراتشخيصى تمركز زدايى از افكار

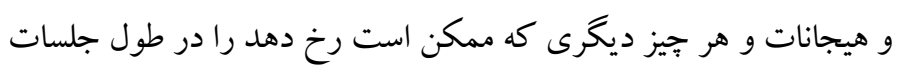
تمرين مى كنند. اينها يكك فرد را قادر مىسازد تا تمر كززدايى ردي را تمرين

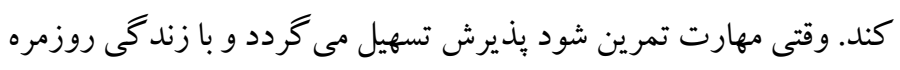

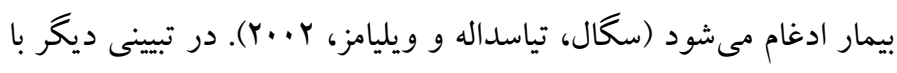
اشاره به اينكه درمان فراتشخيصى مبتنى بر ذهن آكاهى است مى تو أوان تأثير

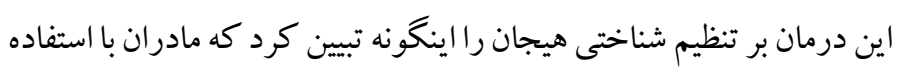

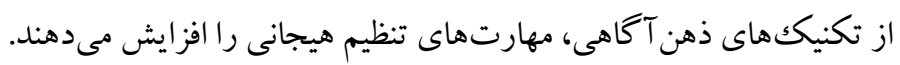
يكى از اين مهارت هاى نظمبخشى هيجانى، ارزيابى مجدد است كه از طريق

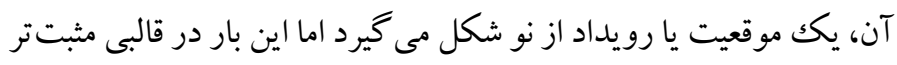

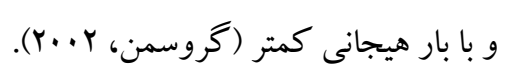

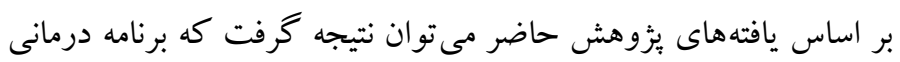

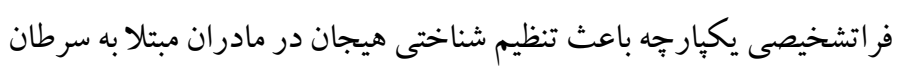

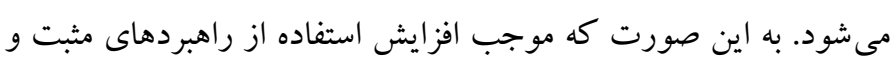

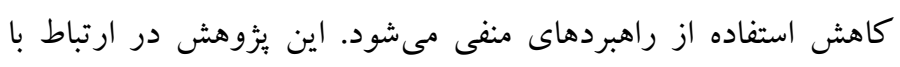
اثربخشى مداخله فرانشخيصى يكهارجهن
تجارب هيجانى داردو وبه واسطه آن يردازش متناسب را تسهيل و ياسخدهى

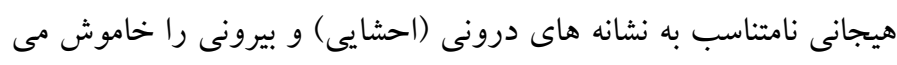
كند. همان طور كه مشخص است اين رويكرد درمانى در بهبود اختلالات هيجانى و تنظيم هيجانات تأثير بسزايى دارد (فاركيونه، الارد، بويسه،

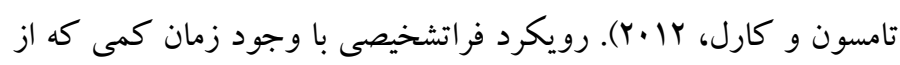

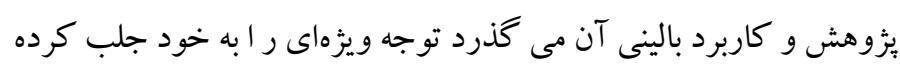

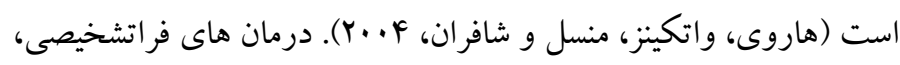

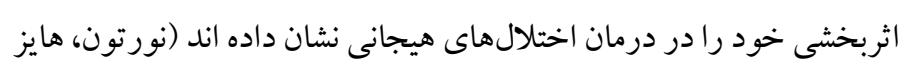

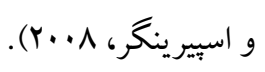

در تبيين يافتهاى اين يُزوهش مى توان به اين موضوع اشاره كرد كه بار

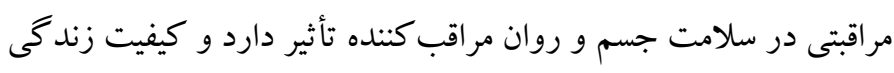
او را تحت تأثير قرار مىدهد. به عبارت ديخر يكك ارتباط مستقيم و معنادار بين كيفيت زندگى كودكك و مادر وجود دارد. بنابراين مطالعات در اين مئن

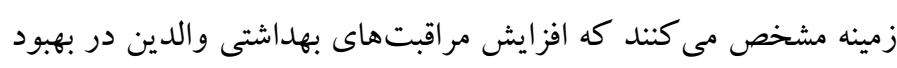

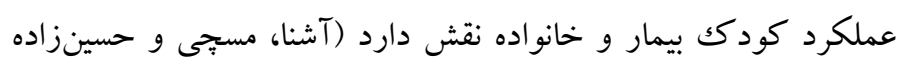

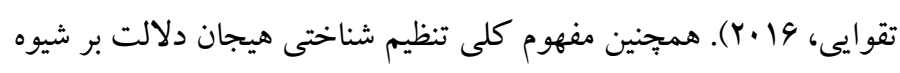

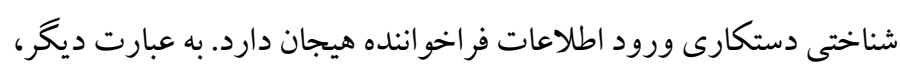

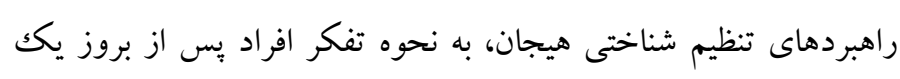

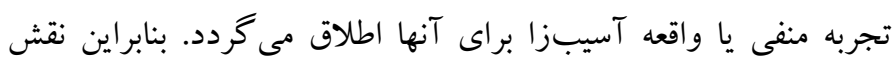

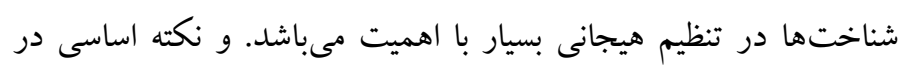

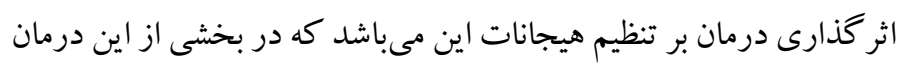

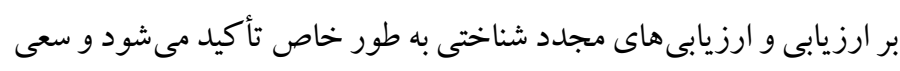

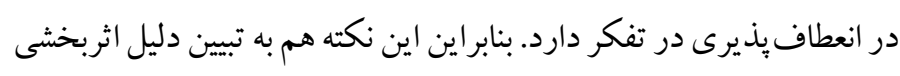

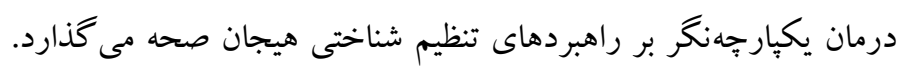
بايد كفت بر واضح است كه اين مادران به دليل محدوديت و مشكلات خود در ارزبابىهاى شناختى و به تبع آن در استفاده صحيح و بجاى وري

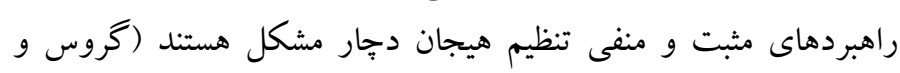

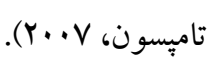

همجنين، با اشاره به اينكه درمان فراتشخيصى با افزايش آكاهى هيجانى

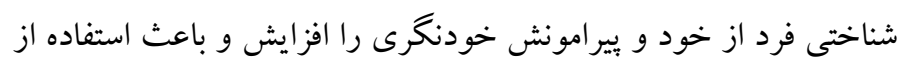

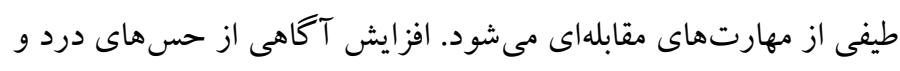

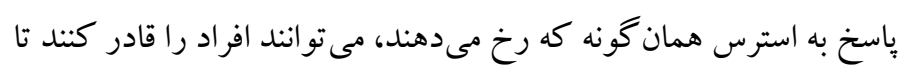


اين مورد اشاره كرد كه اين درمان جديد براى اولين بار در جامعه كود كان

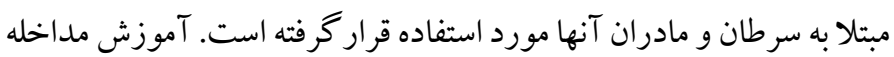

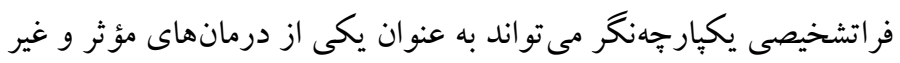

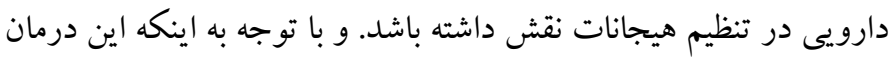

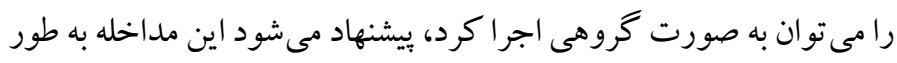

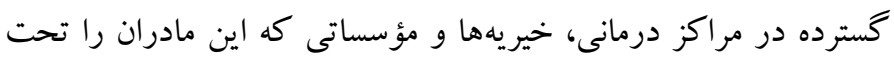

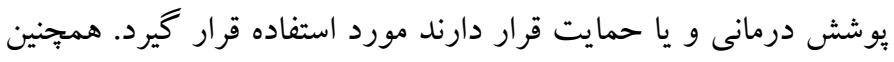
انجام مطالعات بيشتر براى بررسى ساير عومل مؤثر جهت ارتقاى سلامت و

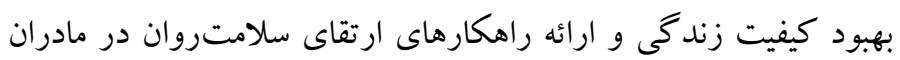
داراى كودك مبتلا به سرطان ضرورى به نظر مىرسد.

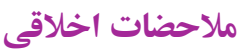

يبيروى از اصول اخلاق بثوهش: ائن مقاله برگرفته از ر ساله دكترى نويسنده اول،

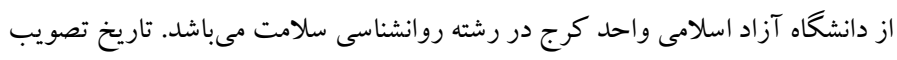

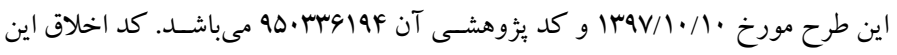

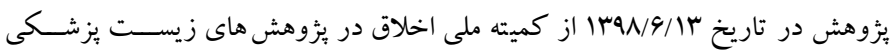
وزارت بهداشــ، درمان و آموزش بزشـكلى به شـماره

اخذ شد.

حامى مالى: اين يزوهش در قالب رساله دكترى و بدون حمايت مالى مىباشد. نقش هر يكك از نويسند كان: نويسنده اول محقق اصلى اين يُزوهش است. نويسنده

دوم استاد راهنما و نويسند كان سوم و جهارم استادان مشاور رساله دكترى مىباشند. تضاد منافع: نو يسند كان هيج تضاد منافعى در رابطه با اين يُوهش اعلام نمىنمايند. تشكر و قدردانى: اين بثوهش، همدلى و همراهى كوجكى با كود كان مبتلا به سرطان

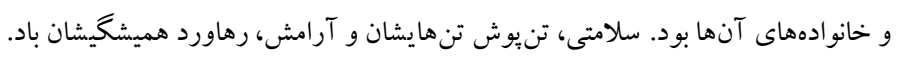

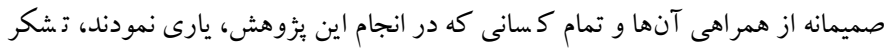
مى كنم.
از معنادارى اثربخشى درمان بر تنظيم هيجانات مادران داراى فرزند مبتلا به

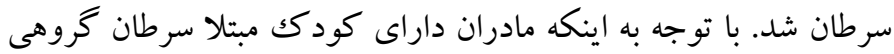

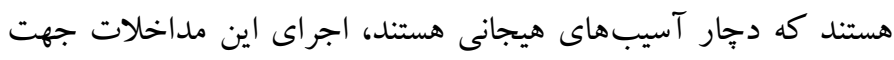

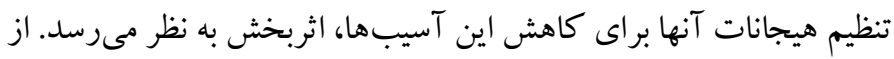
محدوديت هاى يزوهش حاضر، با توجه به شرايط خاص جامعه بزووهش و

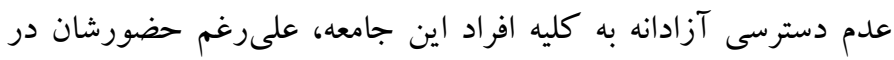

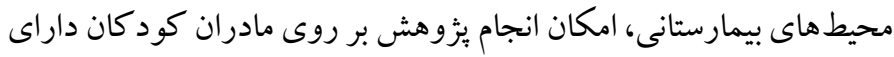
يكك نوع خاص از سرطان وجود نداشت و از اين موضوع و همجينين

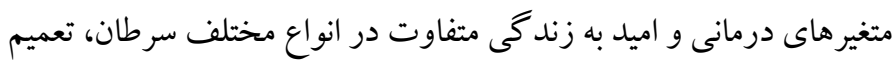

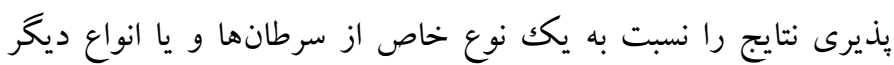
سرطانها كه در اين بزٔوهش جز نمونه نيودند محدود مى كند. از جمله

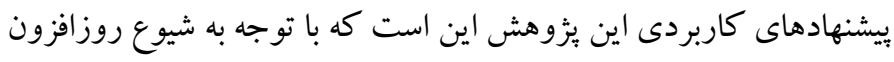

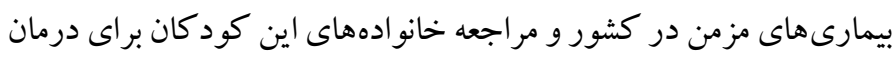
به مراكز درمانى لازم است كه بيمارستانها، كلينيككها و مراكز درمانى مردي

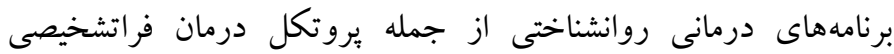

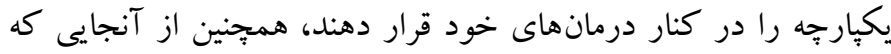
والدين كودكان مبتلا به سرطان مراجعه كننده به بيمارستانها داراى

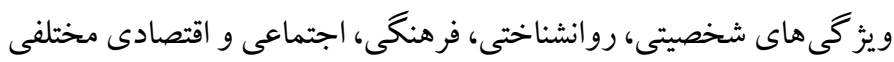

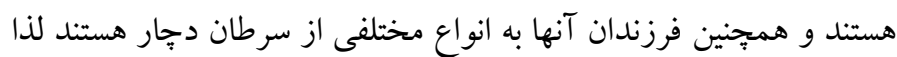

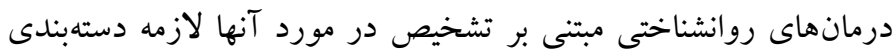
دقيق تر، مر احل و هزينه هاى بيشتر است كه به همين دليل ممكن است مر مراكز

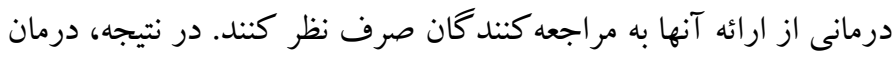

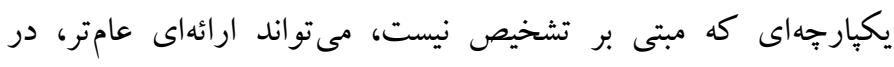

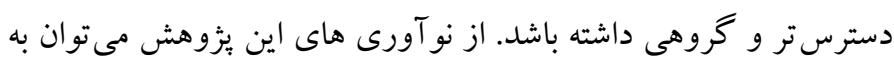




\section{References}

Allen L.B., Mchugh R.K., Barlow D.H. (2007).Emotional disorders: A unified protocol.In:Clinical handbook of psychological disorders: A step-by-step treatment manual. 4th ed. New York: Guilford Press, 216-49. [Link]

Anniko, M,. Fielding, L.B. (2010). A single subject design study testing an emotion-focused transdiagnostic treatment for stress-related ill health. Sweden: Orebro University. [Link]

Ashena M, Besharat M A, Malihialzuckerini S, Rafezi Z. (2020). The effectiveness of unified transdiagnostic therapy on positive metacognitions and metaemotions in mothers of children with cancer. Journal of psychological science, (97)20,. 22-13. (Persian). [Link]

Ashena M, Meschi F, Hosseinzadetaghvayi, M. (2016). The study of the Relationship Between Coping Styles,Acceptance and Action in Parents of CancerStricken Children. The Socail Sciences, 11(14), 3579-3586. [Link]

Ashena M, Meschi F, Hosseinzadetaghvayi, M. (2016). Relationship between Cognitive Emotion Regulation and Acceptance and Action in Parents of Cancer-Stricken Children. International Journal of Advanced Biotechnology and Research (IJBR), 7(2), 629-641. [Link]

Baer, R.A. (2003). Mindfulness training as a clinical intervention: A conceptual and empirical review. Clinical psychology: sience and practice,10,125143. [Link]

Bekui, BA., Aziato,L., Ohene, L.A., Richter, M.S. (2020).Psychological and spiritual wellbeing of family caregivers of children with cancer at a teaching hospital in Ghana. International Journal of Africa Nursing Sciences. (13). [Link]

Besharat, mohammad ali, \& Bazzazian, saeeideh. (2015). Psychometri properties of the Cognitive Emotion Regulation Questionnaire in a sample of Iranian population. Advances in Nursing \& Midwifery, 24(84), 61-70. (Persian). [Link]

Boisseau,C.L.,Farchione,T.J., Fairholme, C. P., Ellard, K. K., Barlow, D. H. (2010). The development of the unified protocol for the transdiagnostic treatment of emotional disorders: A case study. Cognitive and Behavioral Practice, 17(1),102-13. [Link]

Boswell, J.F., Farchione, T.J., Sauer-Zavala, S., Murray, H.W.,Fortune, M.R., Barlow, D.H. (2013). Anxiety sensitivity and interoceptive exposure: A transdiagnostic construct and change strategy. Behavior Therapy, 44(3),417-31. [Link]

Bullis, J.R., Fortune, M.R., Farchione, T.J., Barlow, D.H. (2014). A preliminary investigation of the long-term outcome of the Unified Protocol for Transdiagnostic Treatment of Emotional Disorders. Comprehensive Psychiatry,55,1920-7. [Link]

Castro-Camacho, L., Rattner, M., Quant, D.M., González, L., Moreno, J,D., Ametaj, A. (2019). A Contextual Adaptation of the Unified Protocol for the Transdiagnostic Treatment of Emotional Disorders in Victims of the Armed Conflict in Colombia. Cognitive and Behavioral Practice, 26(2),351-65. [Link]

Doumit M., Rahi, AC., Saab, R., Majdalani, M. (2018).Spirituality among parents of children with cancer in a Middle Eastern country.European Journal of Oncology Nursing,39,21-7. [Link]

Duffy, E., Dias,N., Hendricks-Ferguson, V., Hellsten, M., Skeens-Borland, M. Thornton, C. et al. (2019).Perspectives on Cancer Pain Assessment and Management in Children. Oncology Nursing, 35(3),261-73. [Link]

EmamDoost Z, teymouri S, khoynejad G, rajaei A.(2020).Comparison the effectiveness of mindfulness based cognitive therapy and reality therapy on cognitive emotion regulation in mothers of children with autism spectrum disorders. Journal of psychological science.655-647(89)19. [Link]

Farchione, T.J., Fairholme, C.P., Ellard,K,K., Boisseau, C.L., Thompson-Hollands, J,. Carl, J.R. (2012). Unified protocol for transdiagnostic treatment of emotional disorders: A randomized controlled trial. Behaviour Therapy,43(3),666-78. [Link]

Ghasemzadeh Nasaji S., (2010). Effectiveness of cognitive-behavioral intervention on coping responses and cognitive emotion regulation strategies. International Journal of Behavioral Sciences, 4(1), 35-43. (Persian). [Link]

Grabell, A.S., Huppert,T.J., Fishburn, F.A., Li, Y., Hlutkowsky, C.O., Jones, H.M.,. (2019).Neural correlates of early deliberate emotion regulation: Young children's responses to interpersonal scaffolding. Developmental Cognitive Neuroscience, 40, 2-9. [Link]

Gross,J.J.,Thompson,R.A.(2007).Emotion regulation: Conceptual foundations.Handbook of emotion regulation. New York: Guilford Press,3-24. [Link]

Grossman, D. (2002).Does mindfulness meditation contribute to health? Outcome evaluation of a 
German sample. Journal of alternative \& complementary medicine,8(6),716-30. [Link]

Grootenhuis,M.A.,Last,B.F. (2010).Predictors of parental emotional adjustment to childhood cancer. Psychooncology, (2), 115-28. [Link]

Harvey, A., Watkins, E., Mansell, W., Shafran, R. (2004). Cognitive behavioural processes across psychological disorders: A transdiagnostic approach to research and treatment. Oxford: Oxford University Press. [Link]

Hasanpoor, P., Aghausefi, A., Zamir, O., Alipour, A. (2019). The Effectiveness of Transdiagnostic Treatment on Experiential Avoidance and Cognitive Emotion Regulation in Patients with Obsessive-Compulsive Disorder and its Comparison with Exposure and Prevention Response Therapy. Journal of Clinical Psychology, 11(3), 25-38. (Persian). [Link]

Hoekstra-Weebers, J., Jaspers, J.P., Kamps, W.A., Klip, E.C. (2011). Psychological adaptation and social support of parents of pediatric cancer patients: a prospective longitudinal study. Journal of pediatric psychology,26(4),225-35. [Link]

Hooman F, Mehrabizadeh Honarmand M, Zargar Y, Davodi I. The Effectiveness of Transdiagnostic Therapy on Anxiety, Depression, Cognitive Strategies of Emotional Regulation, and General Performance in Women with Comorbid Anxiety and Depression. JABS. 2016; 5 (4):551-563. (Persian). [Link]

Jamie,A.,Shermanab.J.,Ehrenreich.M. (2019). Changes in Risk Factors during the Unified Protoocol for Transdiagnostic Treatment of Emotional Disorder in Adolescents. Behavior Therapy. Available online 19 December 2019. In press, Journal Pre-proof. [Link]

Jarvisteele, S., Farchione, TJ,. Robbins, CC. Ametaj, A., Sophia, SS., Zavala, D., Barlow, H. (2018).Efficacy of the Unified Protocol for transdiagnostic treatment of comorbid psychopathology accompanying emotional disorders compared to treatments targeting single disorders.Journal of Psychiatric Research, 104,211-216. [Link]

Kadan,G.,Erten,H.,Ogelman,H.G. (2013).The relation between acceptance/ rejection levels perceived by padiatric patients regarding their mothers and acceptance / rejection levels of mothers. Uluslararos Hakemli Aile Couck ve Egitim Dergisi, 1, 104-17. [Link]

kazak, A.E., Alderfer, M.A., Mougianis, I., Barakat, L.P., Beele, D., DiTaranto, S.,Hwang, W.T. (2009).
Family psychosocial risk distress and service utilization in pediatric cancer, Predictive validity oef the Psychosocial Assessment Tool. Cancer, 115, 433-9. [Link]

Klassen, A.F., Raina,P., McIntosh, C., Sung, L., Klaassen,R.J., O'donnell, M. (2011).Parents of children with cancer: which factors explain differences in health-related quality of life. $n t J$ Cancer, 129(5),1190-8. [Link]

Kring, A. M., \& Sloan, D. M. (Eds.). (2009).Emotion regulation and psychopathology: A transdiagnostic approach to etiology and treatment. Guilford Press. [Link]

Kucukoglu, S., Polat, S., Tufekci, F.G., Bulut, H.K. (2016). Acceptance-rejection levels of the Turkish mothers toward their children with cancer. Collegian, 23(2), 217-23. [Link]

Lin, L. (2007). Living with uncertainty: The psychological adjustment and coping by parents of children with cancer in Taiwan. A dissertation submitted to the faculty of the University of North Carolina at Chapel Hill in partial fulfillment of the requirement for the degree of Doctor of Philosophy in the School of Nursing. [Link]

Lu, L., Wang, L.,Yang, X., Feng, Q. (2009). Zarit Caregiver Burden Interview: development, reliability and validity of the Chinese version. Psychiatry and clinical neurosciences,63(6),730-4. [Link]

Maia, A.C., Nardi, A.E., Cardoso, A. (2014).The utilization of unified protocols in behavioral cognitive therapy in transdiagnostic group subjects: A clinical trial. Journal of Affective Disorders, 172, 179-18. [Link]

Majdalani, M,. Doumit, M., Rahi, A.C., Saab, R. (2018). Spirituality among parents of children with cancer in a Middle Eastern country. European Journal of Oncology Nursing, 39,21-7. [Link]

Miller, K.S6 (2010). Factors Associated with Parents' Understanding of their Child's Cancer Prognosis. Dissertation Presented in Partial Fulfillment of the Requirements for the Degree of Doctor of Philosophy. The Ohio State University. [Link]

Nolbris, M., Enska“r., Hellstro“m, A.N,. (2007). Experience of siblings of children treated for cancer. European Journal of Oncology Nursing,11, 106112. [Link]

Norton, P.J., Hayes, S.A., Springer, J.R. (2008). Transdiagnostic, cognitive-behavioral group therapy for anxiety:Outcome and process. 
International Journal of Cognitive Therapy, 1(3), 266-79. [Link]

Sakiris, N.,Berle, D. (2019). A systematic review and meta-analysis of the Unified Protocol as a transdiagnostic emotion regulation based intervention. ClinicalPsychology Review, 72, 101751. [Link]

Schuppert, H.M., Timmerman, M.E., Bloo, J., Gemert, T.G., Wiersema, H.M., Minderaa, R.B., et al. (2012). Emotion Regulation Training for Adolescents With Borderline Personality Disorder Traits: A Randomized Controlled Trial. Journal of the American Academy of Child and Adolescent Psychiatry,51(12),1314-23. [Link]

Segal, Z.V., Teasdale, J.D., Williams, J.M. (2002). Mindfulness Based cognitive therapy for depression.New York. The Guilford Press. [Link]

Varkovitzky, R., Sherrill,A.M., Reger, G. (2018). Effectiveness of the Unified Protocol for Transdiagnostic Treatment of Emotional Disorders Among Veterans With Posttraumatic Stress Disorder: A Pilot Study. Behavior Modification. Behavior Modifiction, 42(2),210-30. [Link]

Vranceanu,A.M.,TalaeiKhoei,M,.NematiRezvani,H.,Fisc herauer,SF.,Ring,D.,Chen,N. (2017). Emotion Regulation Strategies Mediate the Associations of Positive and Negative Affect to Upper Extremity Physical Function. Comprehensive Psychiatry, 72,85-93. [Link]

Yaghoubi H, Vaghef L. (2020). The common and unique role of maladaptive cognitive emotion regulation strategies in predicting emotional problems of nursing students. Journal of psychological science. 722-715(90)19. (Persian). [Link]

Zarit S. H. (2004). Family care and burden at the end of life. CMAJ: Canadian Medical Association journal = journal de l'Association medicale canadienne, 170(12), 1811-1812. [Link]

Zhang, MF., Lyu, QY.,Bu, XQ., Zhou,XZ., Zhao, X. (2019). A qualitative study exploring coping strategies in Chinese families during children's hospitalization for cancer treatment. Journal of Pediatric Nursing, (48),27-34. [Link] 